Journal of Clinical Investigation

Vol. 41, No. 3, 1962

\title{
ASCERTAINING GENETIC CARRIERS OF HEREDITARY SPHEROCYTOSIS BY STATISTICAL ANALYSIS OF MULTIPLE LABORATORY TESTS *
}

\author{
By ARCHIE A. MACKINNEY, JR., $\dagger$ NEWTON E. MORTON, NECHAMA S. KOSOWER \\ AND ROBERT F. SCHILLING
}

\author{
(From the Departments of Medicine and Medical Genetics, University of Wisconsin Medical \\ School, Madison, Wis.)
}

(Submitted for publication August 23, 1961; accepted October 2, 1961)

Large-scale genetic study of hereditary spherocytosis (HS) is complicated by the lack of well defined criteria for diagnosis of mildly affected cases. This paper presents a study of $181 \mathrm{mem}-$ bers of 26 families in which normal members and affected members were classified by analysis of multiple laboratory tests.

Hereditary spherocytosis is a familial hemolytic disease characterized by abnormal thickness of the erythrocyte. The spleen preferentially traps and destroys these abnormally shaped cells $(1,2)$. The hemolysis that results from this normal function of the spleen is terminated by splenectomy. Recent studies have turned from the mechanism of splenic trapping (still unclear) to the cause of sphering. Metabolic defects in the spherocyte were described by Prankerd, Altman and Young (3) in 1955, and recently Prankerd (4) has shown evidence that the metabolic changes are of greater significance to hemolysis than is the shape change. However, the metabolic defects are thought not to be pathognomonic of HS (5). Some evidence has been recently introduced showing that the membrane content of phosphatidyl ethanolamine is decreased (6). To date no unique enzyme defect has been demonstrated. In the absence of a specific diagnostic measurement we are compelled to rely on conventional hematologic procedures including standardized measurements of red cell fragility: osmotic fragility, autohemolysis, and mechanical fragility.

The diagnosis of hereditary spherocytosis can be made with certainty only with laboratory aid and family study. Although some severe cases with clinical jaundice, splenomegaly, anemia, and

\footnotetext{
* Supported by a research grant from the National Institutes of Health.

$\dagger$ Trainee, National Cancer Institute, during part of this study.
}

gall stones have occurred in every series, minimally affected cases have also been encountered, so that as early as 1925 Campbell and Warner (7) cautioned against excluding the disease without laboratory data. Contemporary literature does not make clear the amount of laboratory data required to establish the presence or absence of HS.

Race (8) made the largest of modern family studies 20 years ago but did not describe the laboratory tests used to ascertain his cases. Abrams and Battle (9) appear to have based their genetic conclusions on measurement of red cell diameters, using the Hayden erythrocytometer. Although Young, Izzo and Platzer (10) do not emphasize their method of diagnosis or ascertainment, they state that the mechanical fragility was elevated in every affected case.

Genetic studies of spherocytic families often produce data at variance with the pattern predicted for a single, simple Mendelian dominant. The most frequent abnormality is failure to show 50 per cent incidence in siblings, offspring, and parents of those affected. Young and co-workers (10) found a shortage of affected siblings, which they attributed to low gene penetrance, and a significant number of sporadic cases. In a larger study Race (8) also found a shortage of affected siblings which he attributed to a high incidence of miscarriages and fetal deaths. However, single reports $(9,11)$ of large families may show 50 per cent affected. One genetic study suggests that there is an excess of affected males, an unexpected finding in a simple Mendelian dominant (9). Another genetic study indicates that more than one type of hereditary spherocytosis may exist (12). The reader is referred to Dacie (13) for a comprehensive review.

In this study we have established methods for ascertaining affected and normal subjects in HS 
STATISTICAL DIAGNOSIS OF SPHEROCYTOSIS

TABLE I

Laboratory data of 48 normal controls*

\begin{tabular}{|c|c|c|c|c|c|c|c|c|c|c|c|c|c|c|c|c|c|}
\hline $\begin{array}{c}\text { Family and } \\
\text { Individual } \\
\text { Number }\end{array}$ & $\begin{array}{l}\text { Age } \\
\& \\
\text { Sex }\end{array}$ & 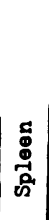 & 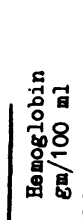 & $\begin{array}{l}\mathrm{MCv} \\
\mathrm{u}^{3}\end{array}$ & $\begin{array}{c}\text { мCH } \\
\mu \mu g\end{array}$ & $\begin{array}{c}\text { MCHC } \\
\%\end{array}$ & 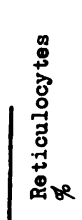 & 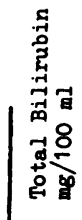 & 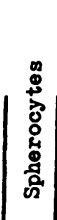 & & $\begin{array}{l}\text { OF } \\
1 \%\end{array}$ & $\begin{array}{l}\text { OF } \\
50 \%\end{array}$ & $\begin{array}{l}\text { AH } \\
\%\end{array}$ & $\underset{\%}{\mathrm{AH}+\mathrm{G}}$ & $\underset{\%}{\mathrm{AH}+\mathrm{Ad}}$ & $\begin{array}{l}\text { Computor } \\
\text { Diagmosis } \\
\text { Dc } D_{4}\end{array}$ & 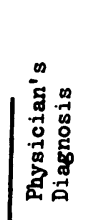 \\
\hline $\begin{array}{r}000-001 \\
002 \\
003 \\
004 \\
005 \\
\end{array}$ & $\begin{array}{l}25 M \\
65 M \\
44 F \\
30 F \\
28 F \\
\end{array}$ & $\begin{array}{l}0 \\
0 \\
0 \\
0 \\
0 \\
\end{array}$ & $\begin{array}{l}16.5 \\
14.3 \\
13.1 \\
11.7 \\
13.3 \\
\end{array}$ & $\begin{array}{l}86 \\
69 \\
79 \\
75 \\
91 \\
\end{array}$ & $\begin{array}{l}29 \\
25 \\
25 \\
22 \\
29 \\
\end{array}$ & $\begin{array}{l}34 \\
38 \\
31 \\
30 \\
32 \\
\end{array}$ & $\begin{array}{l}3.9 \\
3.3 \\
2.4 \\
3.0 \\
3.0 \\
\end{array}$ & $\begin{array}{l}0.8 \\
0.1 \\
0.4 \\
1.0 \\
0.1 \\
\end{array}$ & $\begin{array}{l}1 \\
0 \\
0 \\
0 \\
1 \\
\end{array}$ & $\begin{array}{l}.45 \\
.51 \\
.47 \\
.47 \\
.44 \\
\end{array}$ & $\begin{array}{l}.43 \\
.43 \\
.41 \\
.39 \\
.40 \\
\end{array}$ & $\begin{array}{l}9.6 \\
4.8 \\
3.8 \\
1.1 \\
2.3 \\
\end{array}$ & $\begin{array}{rr}.6 & 4.2 \\
.8 & 5.2 \\
.8 & 2.6 \\
.1 & 11.6 \\
.3 & 2.3 \\
\end{array}$ & $\begin{array}{l}0.2 \\
0.5 \\
0.5 \\
0.4 \\
0.5 \\
\end{array}$ & $\begin{array}{l}1.1 \\
5.2 \\
1.7 \\
1.0 \\
1.4 \\
\end{array}$ & $\begin{array}{l}- \\
\overline{-} \\
\overline{-}\end{array}$ & $\begin{array}{l}\overline{-} \\
\overline{-} \\
\overline{-}\end{array}$ \\
\hline $\begin{array}{l}006 \\
007 \\
009 \\
010 \\
011 \\
\end{array}$ & $\begin{array}{l}33 \mathrm{~F} \\
49 \mathrm{M} \\
73 \mathrm{~F} \\
48 \mathrm{M} \\
32 \mathrm{~F} \\
\end{array}$ & $\begin{array}{l}0 \\
0 \\
0 \\
0 \\
0 \\
\end{array}$ & $\begin{array}{l}14.7 \\
16.0 \\
16.6 \\
15.4 \\
15.0 \\
\end{array}$ & $\begin{array}{r}79 \\
82 \\
103 \\
98 \\
101 \\
\end{array}$ & $\begin{array}{l}26 \\
28 \\
35 \\
33 \\
34 \\
\end{array}$ & $\begin{array}{l}33 \\
34 \\
35 \\
34 \\
33 \\
\end{array}$ & $\begin{array}{l}2.1 \\
2.4 \\
4.4 \\
1.9 \\
4.7 \\
\end{array}$ & $\begin{array}{l}0.3 \\
0.5 \\
0.4 \\
1.0 \\
1.1 \\
\end{array}$ & $\begin{array}{l}1 \\
1 \\
1 \\
1 \\
1 \\
\end{array}$ & $\begin{array}{l}.45 \\
.49 \\
.48 \\
.46 \\
.48 \\
\end{array}$ & $\begin{array}{l}.39 \\
.43 \\
.42 \\
.42 \\
.42 \\
\end{array}$ & $\begin{array}{l}3.1 \\
8.1 \\
4.2 \\
3.2\end{array}$ & $\begin{array}{rr}.1 & 12.0 \\
.1 & 0.8 \\
.3 & 7.0 \\
.4 & 1.1 \\
.2 & 1.9 \\
\end{array}$ & $\begin{array}{l}0.9 \\
0.1 \\
0.6 \\
0.3 \\
0.3 \\
\end{array}$ & $\begin{array}{l}1.2 \\
0.2 \\
1.2 \\
1.0 \\
0.9 \\
\end{array}$ & $\begin{array}{l}- \\
\overline{-} \\
\bar{z} \\
\end{array}$ & $\begin{array}{l}\bar{z} \\
\bar{z} \\
\bar{I}\end{array}$ \\
\hline $\begin{array}{l}013 \\
014 \\
015 \\
016 \\
017\end{array}$ & $\begin{array}{l}21 M \\
63 F \\
39 F \\
29 F \\
27 F\end{array}$ & $\begin{array}{l}0 \\
0 \\
0 \\
0 \\
0\end{array}$ & $\begin{array}{l}15.2 \\
13.5 \\
14.3 \\
14.4 \\
12.8\end{array}$ & $\begin{array}{l}90 \\
89 \\
83 \\
90 \\
80\end{array}$ & $\begin{array}{l}30 \\
28 \\
26 \\
28 \\
25\end{array}$ & $\begin{array}{l}33 \\
33 \\
32 \\
33 \\
31\end{array}$ & $\begin{array}{l}2.1 \\
2.9 \\
2.3 \\
2.3 \\
2.7\end{array}$ & $\begin{array}{l}0.2 \\
0.1 \\
0.2 \\
0.3 \\
0.3\end{array}$ & $\begin{array}{l}0 \\
2 \\
1 \\
0 \\
0\end{array}$ & $\begin{array}{l}.45 \\
.46 \\
.44 \\
.46 \\
.40\end{array}$ & $\begin{array}{l}.39 \\
.44 \\
.38 \\
.39 \\
.36\end{array}$ & $\begin{array}{l}4 . \\
1 . \\
3 . \\
3 . \\
1 .\end{array}$ & $\begin{array}{ll}1 & 5.0 \\
.6 & 3.1 \\
.9 & 1.1 \\
.6 & 1.4 \\
.4 & 0.8 \\
\end{array}$ & $\begin{array}{l}0.5 \\
0.3 \\
0.3 \\
0.3 \\
0.3\end{array}$ & $\begin{array}{l}2.0 \\
1.6 \\
0.8 \\
0.7 \\
0.6\end{array}$ & $\begin{array}{l}\overline{-} \\
\bar{z} \\
\overline{-}\end{array}$ & $\begin{array}{l}\overline{-} \\
\overline{-} \\
\overline{-}\end{array}$ \\
\hline $\begin{array}{l}018 \\
019 \\
020 \\
021 \\
022 \\
\end{array}$ & $\begin{array}{l}66 F \\
23 M \\
20 F \\
34 M \\
61 M \\
\end{array}$ & $\begin{array}{l}0 \\
0 \\
0 \\
0 \\
0 \\
\end{array}$ & $\begin{array}{l}15.6 \\
15.0 \\
14.3 \\
15.7 \\
15.4 \\
\end{array}$ & $\begin{array}{l}86 \\
89 \\
84 \\
90 \\
85 \\
\end{array}$ & $\begin{array}{l}28 \\
32 \\
27 \\
30 \\
27 \\
\end{array}$ & $\begin{array}{l}33 \\
35 \\
36 \\
33 \\
32 \\
\end{array}$ & $\begin{array}{l}3.5 \\
2.7 \\
1.8 \\
3.8 \\
1.6 \\
\end{array}$ & $\begin{array}{l}0.5 \\
0.7 \\
0.5 \\
0.5 \\
0.6 \\
\end{array}$ & $\begin{array}{l}1 \\
2 \\
0 \\
1 \\
0 \\
\end{array}$ & $\begin{array}{l}.45 \\
.45 \\
.44 \\
.48 \\
.46 \\
\end{array}$ & $\begin{array}{l}.40 \\
.39 \\
.37 \\
.43 \\
.42 \\
\end{array}$ & $\begin{array}{l}6.0 \\
7.0 \\
5.0 \\
4.0 \\
4 .\end{array}$ & $\begin{array}{ll}.0 & 1.6 \\
.0 & 3.9 \\
.5 & 3.8 \\
.6 & 2.1 \\
.2 & 2.7 \\
\end{array}$ & $\begin{array}{l}0.2 \\
0.4 \\
0.5 \\
0.4 \\
0.7 \\
\end{array}$ & $\begin{array}{l}0.5 \\
0.7 \\
0.9 \\
1.0 \\
0.8 \\
\end{array}$ & $\begin{array}{l}\overline{-} \\
\overline{-} \\
\overline{-}\end{array}$ & $\begin{array}{l}\bar{z} \\
\bar{z} \\
\bar{z}\end{array}$ \\
\hline $\begin{array}{l}023 \\
024 \\
025 \\
026 \\
027 \\
\end{array}$ & $\begin{array}{l}28 \mathrm{~F} \\
57 \mathrm{M} \\
51 \mathrm{~F} \\
47 \mathrm{~F} \\
32 M \\
\end{array}$ & $\begin{array}{l}0 \\
0 \\
0 \\
0 \\
0 \\
\end{array}$ & $\begin{array}{l}12.9 \\
16.8 \\
12.7 \\
14.5 \\
14.5 \\
\end{array}$ & $\begin{array}{l}89 \\
91 \\
85 \\
78 \\
82 \\
\end{array}$ & $\begin{array}{l}29 \\
31 \\
27 \\
27 \\
26 \\
\end{array}$ & $\begin{array}{l}32 \\
33 \\
32 \\
36 \\
33 \\
\end{array}$ & $\begin{array}{l}2.2 \\
3.4 \\
2.3 \\
1.4 \\
1.4 \\
\end{array}$ & $\begin{array}{l}0.4 \\
1.1 \\
0.2 \\
0.4 \\
0.2 \\
\end{array}$ & $\begin{array}{l}0 \\
1 \\
0 \\
0 \\
0 \\
\end{array}$ & $\begin{array}{l}.45 \\
.46 \\
.49 \\
.49 \\
.47 \\
\end{array}$ & $\begin{array}{l}.37 \\
.43 \\
.43 \\
.43 \\
.41 \\
\end{array}$ & $\begin{array}{l}3 . \\
1 . \\
5 . \\
1.5 \\
2 .\end{array}$ & $\begin{array}{ll}.3 & 9.5 \\
.9 & 0.6 \\
.2 & 2.5 \\
.9 & 5.6 \\
.1 & 0.9 \\
\end{array}$ & $\begin{array}{l}1.4 \\
0.1 \\
0.2 \\
0.6 \\
0.2 \\
\end{array}$ & $\begin{array}{l}2.3 \\
1.9 \\
1.3 \\
0.7 \\
0.7 \\
\end{array}$ & $\begin{array}{l}- \\
\overline{-} \\
\overline{-}\end{array}$ & $\begin{array}{l}\bar{z} \\
\bar{z} \\
\bar{z}\end{array}$ \\
\hline $\begin{array}{l}028 \\
029 \\
030 \\
031 \\
032 \\
\end{array}$ & $\begin{array}{l}25 M \\
33 M \\
30 M \\
33 F \\
25 F \\
\end{array}$ & $\begin{array}{l}0 \\
0 \\
0 \\
0 \\
0 \\
\end{array}$ & $\begin{array}{l}12.9 \\
14.5 \\
16.8 \\
15.8 \\
13.0 \\
\end{array}$ & $\begin{array}{l}84 \\
86 \\
86 \\
88 \\
90 \\
\end{array}$ & $\begin{array}{l}27 \\
28 \\
31 \\
30 \\
31 \\
\end{array}$ & $\begin{array}{l}33 \\
33 \\
33 \\
34 \\
32 \\
\end{array}$ & $\begin{array}{l}2.7 \\
3.9 \\
2.6 \\
2.9 \\
1.7 \\
\end{array}$ & $\begin{array}{l}0.2 \\
1.2 \\
0.1 \\
0.4 \\
1.3 \\
\end{array}$ & $\begin{array}{l}0 \\
0 \\
0 \\
0 \\
0 \\
\end{array}$ & $\begin{array}{l}.45 \\
.46 \\
.45 \\
.47 \\
.46 \\
\end{array}$ & $\begin{array}{l}.38 \\
.39 \\
.40 \\
.42 \\
.39 \\
\end{array}$ & $\begin{array}{l}5 . \\
4 . \\
4.8 \\
2 . \\
4.0\end{array}$ & $\begin{array}{rr}1 & 1.6 \\
.7 & 30.3 \\
.8 & 10.2 \\
.1 & 2.4 \\
.0 & 3.3 \\
\end{array}$ & $\begin{array}{l}0.1 \\
1.0 \\
1.5 \\
0.1 \\
0.1 \\
\end{array}$ & $\begin{array}{l}0.7 \\
7.4 \\
6.2 \\
0.5 \\
2.2 \\
\end{array}$ & $\begin{array}{l}\overline{-} \\
\overline{-} \\
\overline{-}\end{array}$ & $\begin{array}{l}\bar{z} \\
\bar{z} \\
\bar{z}\end{array}$ \\
\hline $\begin{array}{r}033 \\
000-034 \\
035 \\
036 \\
037 \\
038 \\
\end{array}$ & $\begin{array}{l}53 F \\
27 F \\
26 F \\
28 X \\
19 K \\
20 M \\
\end{array}$ & $\begin{array}{l}0 \\
0 \\
0 \\
0 \\
0 \\
0 \\
\end{array}$ & $\begin{array}{l}11.9 \\
13.9 \\
13.9 \\
13.3 \\
15.4 \\
14.8 \\
\end{array}$ & $\begin{array}{l}91 \\
89 \\
98 \\
88 \\
96 \\
97 \\
\end{array}$ & $\begin{array}{l}32 \\
29 \\
32 \\
30 \\
31 \\
32 \\
\end{array}$ & $\begin{array}{l}29 \\
32 \\
32 \\
33 \\
32 \\
33 \\
\end{array}$ & $\begin{array}{l}2.6 \\
2.5 \\
2.4 \\
2.0 \\
2.6 \\
2.2 \\
\end{array}$ & $\begin{array}{l}0.3 \\
0.6 \\
0.3 \\
0.3 \\
0.8 \\
0.2 \\
\end{array}$ & $\begin{array}{l}0 \\
0 \\
0 \\
0 \\
0 \\
0 \\
\end{array}$ & $\begin{array}{l}.46 \\
.47 \\
.45 \\
.46 \\
.44 \\
.51 \\
\end{array}$ & $\begin{array}{l}.39 \\
.41 \\
.39 \\
.40 \\
.38 \\
.44 \\
\end{array}$ & $\begin{array}{r}2.1 \\
4.7 \\
4.8 \\
5.0 \\
4.4 \\
4.6 \\
\end{array}$ & $\begin{array}{c}1.8 \\
2.4 \\
2.0 \\
2.1 \\
10.6 \\
12.3 \\
\end{array}$ & $\begin{array}{l}0.2 \\
0.1 \\
0.5 \\
0.2 \\
0.5 \\
0.7 \\
\end{array}$ & $\begin{array}{l}\quad 0.8 \\
0.8 \\
0.9 \\
0.7 \\
2.5 \\
2.0 \\
\end{array}$ & $\begin{array}{ll}- & - \\
- & - \\
- & - \\
- & - \\
- & -\end{array}$ & $\begin{array}{l}- \\
= \\
= \\
=\end{array}$ \\
\hline $\begin{array}{l}039 \\
040 \\
041 \\
042 \\
043 \\
\end{array}$ & $\begin{array}{l}22 x \\
24 x \\
23 x \\
43 F \\
23 x \\
\end{array}$ & $\begin{array}{l}0 \\
0 \\
0 \\
0 \\
0 \\
\end{array}$ & $\begin{array}{l}15.2 \\
15.6 \\
13.91 \\
11.81 \\
16.61 \\
\end{array}$ & $\begin{array}{r}88 \\
98 \\
105 \\
116 \\
106 \\
\end{array}$ & $\begin{array}{l}30 \\
31 \\
34 \\
37 \\
34 \\
\end{array}$ & $\begin{array}{l}34 \\
31 \\
32 \\
32 \\
32 \\
\end{array}$ & $\begin{array}{l}2.2 \\
2.2 \\
3.7 \\
1.9 \\
3.0 \\
\end{array}$ & $\begin{array}{l}0.6 \\
0.9 \\
0.3 \\
0.4 \\
1.1 \\
\end{array}$ & $\begin{array}{l}0 \\
0 \\
0 \\
1 \\
1 \\
\end{array}$ & $\begin{array}{l}.45 \\
.45 \\
.44 \\
.45 \\
.46 \\
\end{array}$ & $\begin{array}{l}.38 \\
.38 \\
.39 \\
.41 \\
.41 \\
\end{array}$ & $\begin{array}{l}5.1 \\
3.5 \\
3.5 \\
0.7 \\
3.3 \\
\end{array}$ & $\begin{array}{r}10.1 \\
10.2 \\
10.3 \\
7.7 \\
6.7 \\
\end{array}$ & $\begin{array}{l}0.7 \\
0.8 \\
0.9 \\
0.6 \\
1.2 \\
\end{array}$ & $\begin{array}{l}4.7 \\
1.4 \\
1.4 \\
4.0 \\
1.7 \\
\end{array}$ & $\begin{array}{l}\bar{z} \\
\bar{z} \\
\overline{-}\end{array}$ & $\begin{array}{l}\overline{-} \\
\overline{-} \\
\overline{-}\end{array}$ \\
\hline $\begin{array}{l}044 \\
045 \\
046 \\
048 \\
049 \\
\end{array}$ & $\begin{array}{l}28 M \\
29 F \\
20 F \\
32 F \\
40 X \\
\end{array}$ & $\begin{array}{l}0 \\
0 \\
0 \\
0 \\
0 \\
\end{array}$ & $\begin{array}{l}16.0 \\
13.9 \\
13.5 \\
14.3 \\
15.4 \\
\end{array}$ & $\begin{array}{r}87 \\
104 \\
68 \\
90 \\
86 \\
\end{array}$ & $\begin{array}{l}30 \\
33 \\
29 \\
30 \\
29 \\
\end{array}$ & $\begin{array}{l}34 \\
32 \\
32 \\
31 \\
33 \\
\end{array}$ & $\begin{array}{l}3.1 \\
2.8 \\
1.5 \\
1.4 \\
3.6 \\
\end{array}$ & $\begin{array}{l}0.4 \\
0.6 \\
0.5 \\
c .1 \\
0.5 \\
\end{array}$ & $\begin{array}{l}2 \\
0 \\
0 \\
0 \\
0 \\
\end{array}$ & $\begin{array}{l}.46 \\
.46 \\
.46 \\
.46 \\
.48 \\
\end{array}$ & $\begin{array}{l}.40 \\
.41 \\
.43 \\
.38 \\
.41 \\
\end{array}$ & $\begin{array}{l}7.3 \\
4.4 \\
2.2 \\
2.0 \\
6.5 \\
\end{array}$ & $\begin{array}{r}16.2 \\
19.0 \\
10.6 \\
2.4 \\
1.6 \\
\end{array}$ & $\begin{array}{l}0.7 \\
0.8 \\
0.8 \\
0.5 \\
0.3 \\
\end{array}$ & $\begin{array}{l}2.3 \\
6.8 \\
1.8 \\
1.6 \\
0.8 \\
\end{array}$ & $\begin{array}{l}\overline{-} \\
\overline{-} \\
\overline{-}\end{array}$ & $\begin{array}{l}\bar{z} \\
\bar{z} \\
\overline{-}\end{array}$ \\
\hline $\begin{array}{l}050 \\
051\end{array}$ & $\begin{array}{l}69 \mathrm{~F} \\
38 \mathrm{M}\end{array}$ & $\begin{array}{l}0 \\
0\end{array}$ & $\begin{array}{l}14.61 \\
15.9\end{array}$ & $\begin{array}{r}105 \\
79\end{array}$ & $\begin{array}{l}35 \\
27\end{array}$ & $\begin{array}{l}33 \\
34\end{array}$ & $\begin{array}{l}2.5 \\
2.7\end{array}$ & $\begin{array}{l}0.5 \\
0.9\end{array}$ & $\begin{array}{l}0 \\
0\end{array}$ & $\begin{array}{l}.47 \\
.45\end{array}$ & $\begin{array}{l}.42 \\
.41\end{array}$ & $\begin{array}{l}5.6 \\
3.3\end{array}$ & $\begin{array}{r}26.4 \\
4.3\end{array}$ & $\begin{array}{l}1.1 \\
0.5\end{array}$ & $\begin{array}{l}3.0 \\
1.2\end{array}$ & $\overline{-}$ & $\overline{-}$ \\
\hline Mean & & & 14.5 & 892 & $29.6^{\circ}$ & 33 & 2.6 & 0.5 & & .46 & $.4 !$ & 4.1 & 6.1 & 05 & 1.8 & & \\
\hline Standard $D$ & eviati & & 1.31 & 9.5 & 3.0 & 1.4 & 0.8 & 0.3 & & .02 & .02 & 1.8 & 6.5. & 1.1 & 1.7 & & \\
\hline
\end{tabular}

* Spleen 0 indicates spleen not examined. Spherocyte scores are rated $0-3$ according to the number of examiners who found spherocytes. $\mathrm{MCV}=$ mean corpuscular volume; $\mathrm{MCH}=$ mean corpuscular hemoglobin; $\mathrm{MCHC}=$ mean corpuscular hemoglobin concentration. OF $1 \%$ and $\mathrm{OF} 50 \%$ are interpolated values of osmotic fragility corresponding to the saline tonicities listed. $\mathrm{MF}=$ mechanical fragility; $\mathrm{AH}=$ autohemolysis; $\mathrm{AH}+\mathrm{G}=$ autohemolysis with glucose added; $\mathrm{AH}+\mathrm{Ad}=$ autohemolysis with adenosine added; $\mathrm{Dc}$ and $\mathrm{D}_{4}$ are discriminants (see text) ; $-=$ normal.

families, using Fisher's discriminant function. sis of multiple laboratory tests would give better We have tested the value of combinations of he- genetic data than was previously possible by dematologic measurements for diagnosis. We have creasing the overlap between normal and affected also considered the possibility that statistical analy- and consequently increasing the number of mildly 
affected cases ascertained. A brief resume of genetic conclusions will be given. More elaborate genetic analysis and detailed clinical description are subjects of subsequent papers $(14,15)$.

\section{METHODS}

1. Collection of cases. Letters requesting family study were sent to patients previously diagnosed in this medical center; 181 members of 26 families were investigated in detail. Seven of these families were found during the 4-year period of study. Other family members (in addition to the 181 recorded here) were investigated too late to be included in the statistical study. We attempted to study all siblings in order to avoid bias. Controls were volunteers from the hospital staff and normal spouses of family members.

2. Examination. Each person was examined by history, hematologic tests and, in most cases, palpation of the abdomen for spleen size. Patients were interviewed specifically for information about splenic enlargement and splenectomy, as well as gall bladder disease, jaundice, anemia, and leg ulcers. The question of congenital anomalies was raised without specifying abnormalities.

3. Collection of samples. Fasting blood specimens were not practical because of long distances traveled by patients. Samples of blood were drawn into double oxalate (16) for routine blood and reticulocyte counts. Blood for fragility studies was defibrinated with glass beads.

4. Laboratory methods. Erythrocyte and leukocyte counts were done by conventional techniques using U. S. Bureau of Standards certified hemocytometers and pipets. Coverslip smears from controls and all family members were stained with Kingsley's reagent, given a code number, and examined for spherocytes by three experienced observers; slides were graded 0-3 according to the number of observers who found spherocytes. Reticulocyte percentages were determined by counting 1,000 cells stained with brilliant cresyl blue. ${ }^{1}$ More than 90 per cent of the erythrocyte and reticulocyte counts were done by the same technician. Hemoglobin was measured colorimetrically as cyanmethemoglobin. Serum bilirubin was done by the method of Ducci and Wat-

1 Higher values are obtained in this laboratory with brilliant cresyl blue than with the new methylene blue.

TABLE II

Laboratory data of 87 unaffected family members with spleens intact*

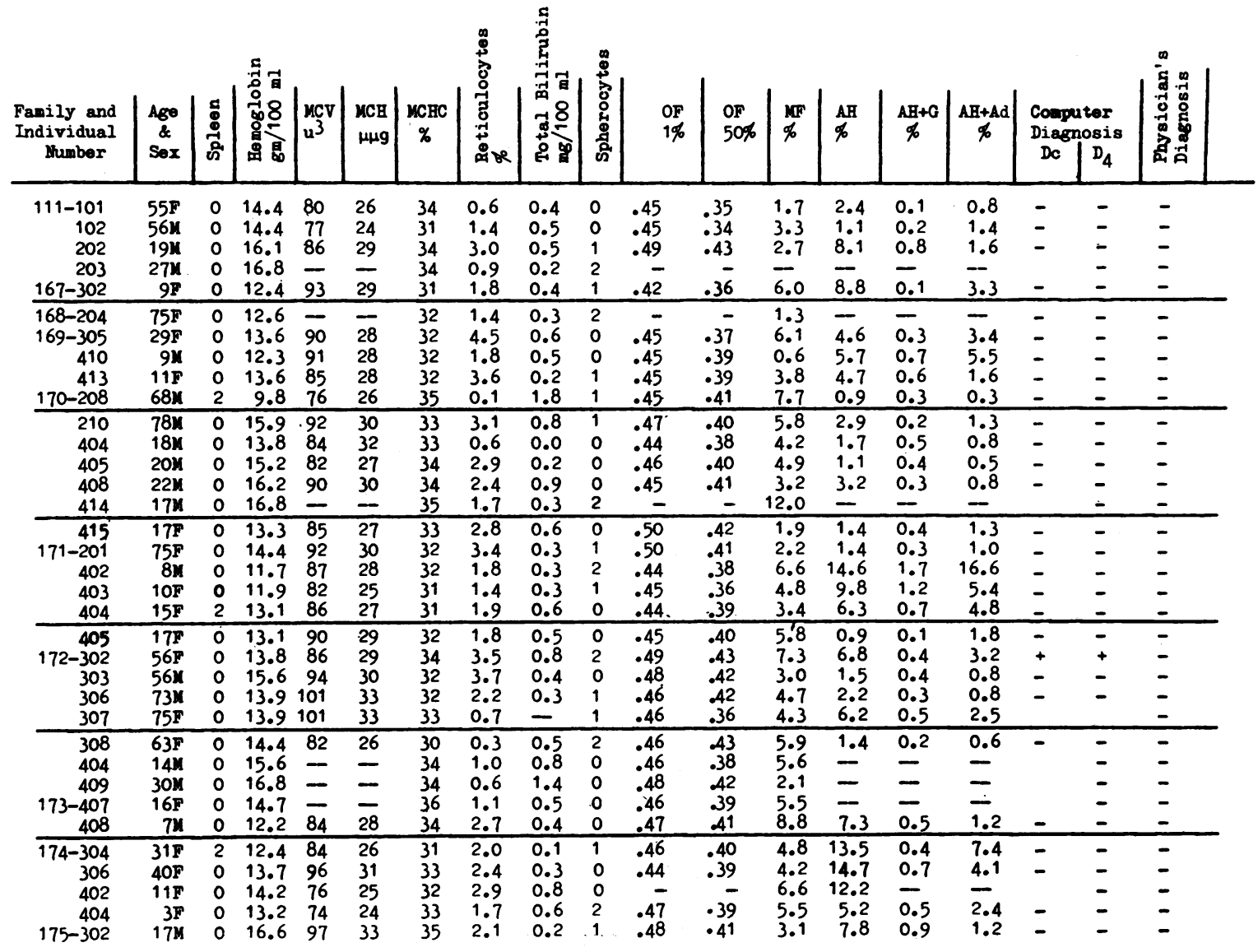




\begin{tabular}{|c|c|c|c|c|c|c|c|c|c|c|c|c|c|c|c|c|c|}
\hline $\begin{array}{c}\text { Family and } \\
\text { Individual } \\
\text { Number }\end{array}$ & $\begin{array}{l}\text { Age } \\
\& \\
\text { Sex }\end{array}$ & 怘 & 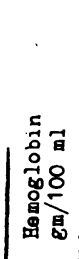 & $\mid \begin{array}{l}\mathrm{mcv} \\
\mathrm{u}^{3}\end{array}$ & $\begin{array}{r}\text { MCH } \\
\mu \mu g\end{array}$ & $\begin{array}{c}\text { MCBC } \\
\%\end{array}$ & 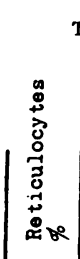 & 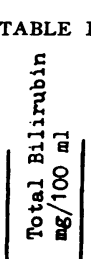 & 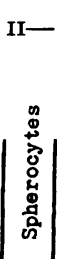 & $\begin{array}{l}\text { OF } \\
1 \%\end{array}$ & $\begin{array}{l}\text { of } \\
50 \%\end{array}$ & $\frac{M F}{\%}$ & $\stackrel{\mathrm{AH}}{\%}$ & $\underset{\%}{\mathrm{AH}+\mathrm{G}}$ & $\underset{\%}{A \mathrm{~A}+\mathrm{Ad}}$ & $\begin{array}{l}\text { Computer } \\
\text { Diasnosis } \\
\text { Dc } \mid D_{4}\end{array}$ & 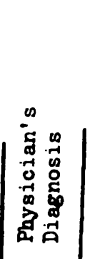 \\
\hline $\begin{array}{r}175-303 \\
304 \\
176-304 \\
305 \\
306 \\
\end{array}$ & $\begin{array}{r}14 M \\
7 M \\
45 F \\
51 F \\
39 M \\
\end{array}$ & $\begin{array}{l}0 \\
0 \\
0 \\
0 \\
0 \\
\end{array}$ & $\begin{array}{l}13.9 \\
12.5 \\
13.1 \\
12.4 \\
15.0 \\
\end{array}$ & $\begin{array}{l}\frac{78}{91} \\
93 \\
83 \\
83\end{array}$ & $\begin{array}{l}26 \\
\frac{31}{27} \\
28 \\
\end{array}$ & $\begin{array}{l}33 \\
33 \\
34 \\
33 \\
34 \\
\end{array}$ & $\begin{array}{l}1.8 \\
0.3 \\
2.7 \\
2.7 \\
2.5 \\
\end{array}$ & $\begin{array}{l}0.6 \\
0.5 \\
0.2 \\
0.6 \\
0.4 \\
\end{array}$ & $\begin{array}{l}1 \\
1 \\
0 \\
1 \\
1 \\
\end{array}$ & $\begin{array}{l}.44 \\
.47 \\
.49 \\
.49 \\
\end{array}$ & $\begin{array}{l}.38 \\
.42 \\
.42 \\
.42 \\
\end{array}$ & $\begin{array}{l}2.7 \\
4.9 \\
3.3 \\
1.3 \\
7.6 \\
\end{array}$ & $\begin{array}{l}1.7 \\
\frac{1.3}{1.2} \\
3.6 \\
\end{array}$ & $\begin{array}{l}0.2 \\
0.3 \\
0.2 \\
0.4 \\
\end{array}$ & $\begin{array}{l}0.6 \\
0.8 \\
0.8 \\
1.0\end{array}$ & $\begin{array}{l}z \\
z \\
z\end{array}$ & $\begin{array}{l}z \\
z \\
z\end{array}$ \\
\hline $\begin{array}{l}308 \\
403 \\
404 \\
405 \\
409\end{array}$ & $\begin{array}{r}62 F \\
17 M \\
8 M \\
10 M \\
2 F\end{array}$ & $\begin{array}{l}0 \\
0 \\
0 \\
0 \\
0\end{array}$ & $\begin{array}{l}15.0 \\
14.4 \\
11.9 \\
12.0 \\
12.3\end{array}$ & $\begin{array}{l}97 \\
88 \\
80 \\
80 \\
-\end{array}$ & $\begin{array}{l}32 \\
27 \\
24 \\
25 \\
25\end{array}$ & $\begin{array}{l}33 \\
31 \\
33 \\
31 \\
-\end{array}$ & $\begin{array}{l}2.9 \\
2.6 \\
2.1 \\
4.2 \\
1.7\end{array}$ & $\begin{array}{l}0.4 \\
0.5 \\
0.5 \\
0.2 \\
--\end{array}$ & $\begin{array}{l}0 \\
1 \\
1 \\
1 \\
0\end{array}$ & $\begin{array}{l}.51 \\
.48 \\
.45 \\
.47 \\
-\end{array}$ & $\begin{array}{l}.44 \\
.41 \\
.39 \\
.40 \\
-\end{array}$ & $\begin{array}{l}2.8 \\
4.3 \\
4.2 \\
5.1 \\
-\end{array}$ & $\begin{array}{l}4.2 \\
9.6 \\
6.4 \\
1.9 \\
-\end{array}$ & $\begin{array}{l}0.4 \\
0.2 \\
0.5 \\
0.1 \\
-\end{array}$ & $\begin{array}{l}1.6 \\
2.6 \\
2.8 \\
0.1 \\
-\end{array}$ & $\begin{array}{l}- \\
\overline{-} \\
-\end{array}$ & $\begin{array}{l}\bar{z} \\
\bar{z}\end{array}$ \\
\hline $\begin{array}{l}410 \\
411 \\
412 \\
413 \\
414 \\
\end{array}$ & $\begin{array}{l}16 \mathrm{~K} \\
15 \mathrm{~F} \\
11 \mathrm{M} \\
10 \mathrm{~F} \\
8 \mathrm{~F} \\
\end{array}$ & $\begin{array}{l}0 \\
0 \\
0 \\
0 \\
0 \\
\end{array}$ & $\begin{array}{l}14.8 \\
13.5 \\
13.5 \\
13.9 \\
13.2 \\
\end{array}$ & $\begin{array}{l}95 \\
89 \\
79 \\
83 \\
78 \\
\end{array}$ & $\begin{array}{l}31 \\
29 \\
27 \\
28 \\
27 \\
\end{array}$ & $\begin{array}{l}32 \\
33 \\
34 \\
33 \\
34 \\
\end{array}$ & $\begin{array}{l}2.9 \\
3.2 \\
3.1 \\
1.9 \\
0.8 \\
\end{array}$ & $\begin{array}{l}0.4 \\
0.1 \\
0.1 \\
0.0 \\
0.1 \\
\end{array}$ & $\begin{array}{l}1 \\
1 \\
0 \\
0 \\
0 \\
\end{array}$ & $\begin{array}{l}.49 \\
.48 \\
.48 \\
.46 \\
.48 \\
\end{array}$ & $\begin{array}{l}.43 \\
.41 \\
.41 \\
.40 \\
.42 \\
\end{array}$ & $\begin{array}{l}7.0 \\
5.6 \\
6.8 \\
4.5 \\
6.5 \\
\end{array}$ & $\begin{array}{l}3.4 \\
1.4 \\
1.4 \\
0.8 \\
2.3 \\
\end{array}$ & $\begin{array}{l}0.5 \\
0.6 \\
0.4 \\
0.5 \\
0.4 \\
\end{array}$ & $\begin{array}{l}1.5 \\
0.7 \\
0.8 \\
0.7 \\
1.1 \\
\end{array}$ & $\begin{array}{l}- \\
\bar{z} \\
- \\
-\end{array}$ & $\begin{array}{l}- \\
z \\
z\end{array}$ \\
\hline $\begin{array}{l}415 \\
416 \\
417 \\
418 \\
419 \\
\end{array}$ & $\begin{array}{r}5 M \\
3 M \\
10 M \\
6 F \\
4 M \\
\end{array}$ & $\begin{array}{l}0 \\
2 \\
2 \\
0 \\
0 \\
\end{array}$ & $\begin{array}{l}12.3 \\
12.5 \\
12.8 \\
12.3 \\
12.4 \\
\end{array}$ & $\begin{array}{l}76 \\
77 \\
84 \\
92 \\
89 \\
\end{array}$ & $\begin{array}{l}26 \\
27 \\
27 \\
30 \\
31 \\
\end{array}$ & $\begin{array}{l}34 \\
34 \\
34 \\
32 \\
35 \\
\end{array}$ & $\begin{array}{l}3.0 \\
2.4 \\
2.3 \\
3.3 \\
2.8 \\
\end{array}$ & $\begin{array}{l}0.0 \\
0.1 \\
0.5 \\
0.3 \\
0.3 \\
\end{array}$ & $\begin{array}{l}1 \\
1 \\
1 \\
1 \\
2 \\
\end{array}$ & $\begin{array}{r}- \\
- \\
.45 \\
.46 \\
.50 \\
\end{array}$ & $\begin{array}{r}- \\
. \\
.40 \\
.40 \\
.43 \\
\end{array}$ & $\begin{array}{l}4.1 \\
4.0 \\
8.7 \\
7.3 \\
3.5 \\
\end{array}$ & $\begin{array}{l}1.4 \\
3.0 \\
1.3 \\
2.5 \\
3.1 \\
\end{array}$ & $\begin{array}{l}\bar{z} \\
\overline{0} \\
0.3\end{array}$ & $\begin{array}{l}\bar{z} \\
\bar{z} \\
1.0\end{array}$ & $\begin{array}{l}- \\
\overline{-} \\
\overline{+}\end{array}$ & $\begin{array}{l}\overline{-} \\
\overline{-} \\
\overline{-}\end{array}$ \\
\hline $\begin{array}{r}422 \\
426 \\
503 \\
177-305 \\
401 \\
\end{array}$ & $\begin{array}{r}29 M \\
38 M \\
2 K \\
32 F \\
1 M \\
\end{array}$ & $\begin{array}{l}0 \\
0 \\
0 \\
0 \\
0 \\
\end{array}$ & $\begin{array}{l}12.6 \\
15.0 \\
11.6 \\
14.3 \\
12.0 \\
\end{array}$ & $\begin{array}{r}98 \\
89 \\
79 \\
91 \\
-\end{array}$ & $\begin{array}{l}32 \\
29 \\
25 \\
31 \\
25 \\
\end{array}$ & $\begin{array}{r}32 \\
33 \\
32 \\
34 \\
\end{array}$ & $\begin{array}{l}3.7 \\
1.7 \\
2.0 \\
1.4 \\
3.4 \\
\end{array}$ & $\begin{array}{l}0.3 \\
0.1 \\
0.2 \\
0.4 \\
- \\
\end{array}$ & $\begin{array}{l}0 \\
0 \\
0 \\
0 \\
0 \\
\end{array}$ & $\begin{array}{r}.52 \\
.53 \\
.56 \\
.46 \\
\end{array}$ & $\begin{array}{r}.45 \\
.46 \\
-39 \\
.39 \\
\end{array}$ & $\begin{array}{l}3.6 \\
4.3 \\
5.6 \\
2.7 \\
- \\
\end{array}$ & $\begin{array}{l}1.9 \\
4.5 \\
\frac{1.0}{1.0} \\
\end{array}$ & $\begin{array}{l}0.4 \\
1.1 \\
0.4 \\
-\end{array}$ & $\begin{array}{l}1.2 \\
1.7 \\
0.7 \\
-\end{array}$ & $\begin{array}{ll}- & - \\
- & - \\
- & -\end{array}$ & $\begin{array}{l}\bar{z} \\
\bar{z}\end{array}$ \\
\hline $\begin{array}{r}402 \\
403 \\
178-501 \\
179-203 \\
180-302\end{array}$ & $\begin{array}{r}2 M \\
1 F \\
3 M \\
34 F \\
48 M \\
\end{array}$ & $\begin{array}{l}0 \\
0 \\
0 \\
0 \\
0\end{array}$ & $\begin{array}{l}12.0 \\
10.9 \\
12.5 \\
12.3 \\
16.1\end{array}$ & $\begin{array}{l}\overline{-} \\
\overline{90} \\
79 \\
95\end{array}$ & $\begin{array}{l}25 \\
25 \\
30 \\
24 \\
33\end{array}$ & $\begin{array}{l}\overline{-} \\
34 \\
31- \\
34\end{array}$ & $\begin{array}{l}2.5 \\
2.8 \\
2.0 \\
1.7 \\
2.8\end{array}$ & $\begin{array}{l}1.0 \\
0.0 \\
0.2 \\
0.4 \\
0.5\end{array}$ & $\begin{array}{l}1 \\
1 \\
0 \\
0 \\
0\end{array}$ & $\begin{array}{l}- \\
. \overline{-} \\
.49 \\
.42\end{array}$ & $\begin{array}{r}- \\
. \\
.37 \\
.40 \\
.47\end{array}$ & $\begin{array}{l}3.9 \\
- \\
3.9 \\
3.9 \\
4.9\end{array}$ & $\begin{array}{l}\overline{-} \\
2.7 \\
5.4 \\
3.2\end{array}$ & $\begin{array}{l}\overline{-} \\
0.1 \\
1.2 \\
0.2\end{array}$ & $\begin{array}{l}\overline{-} \\
0.6 \\
1.9 \\
0.8\end{array}$ & $\begin{array}{l}\bar{z} \\
\bar{z} \\
\bar{z}\end{array}$ & $\begin{array}{l}\bar{z} \\
\bar{z} \\
\bar{z}\end{array}$ \\
\hline $\begin{array}{r}181-202 \\
182-308 \\
401 \\
403 \\
183-201\end{array}$ & $\begin{array}{r}28 \mathrm{~F} \\
26 M \\
19 F \\
6 \mathrm{~F} \\
38 \mathrm{M}\end{array}$ & $\begin{array}{l}0 \\
0 \\
0 \\
0 \\
0\end{array}$ & $\begin{array}{l}13.1 \\
14.4 \\
13.2 \\
13.9 \\
17.0\end{array}$ & $\begin{array}{l}78 \\
\frac{85}{76}\end{array}$ & $\frac{30}{27}$ & $\begin{array}{l}33 \\
35 \\
35 \\
34 \\
36\end{array}$ & $\begin{array}{l}1.3 \\
2.2 \\
1.0 \\
1.1 \\
1.8\end{array}$ & $\begin{array}{l}0.2 \\
0.6 \\
0.3 \\
0.2 \\
0.8\end{array}$ & $\begin{array}{l}2 \\
3 \\
3 \\
3 \\
1 \\
0\end{array}$ & $\begin{array}{r}.48 \\
.47 \\
- \\
.46\end{array}$ & $\begin{array}{r}.44 \\
.43 \\
- \\
.40\end{array}$ & $\begin{array}{l}6.4 \\
8.1 \\
8.1 \\
5.4 \\
4.2\end{array}$ & $\begin{array}{l}5.7 \\
8.2 \\
= \\
\overline{1.8}\end{array}$ & $\begin{array}{l}0.7 \\
0.6 \\
= \\
0.2\end{array}$ & $\begin{array}{l}0.8 \\
2.5 \\
= \\
=\end{array}$ & $\begin{array}{rr}- & - \\
+ & + \\
- & - \\
-\end{array}$ & $\begin{array}{l}\bar{z} \\
\bar{z} \\
\overline{-}\end{array}$ \\
\hline $\begin{array}{c}\text { Family and } \\
\text { Individual } \\
\text { Number }\end{array}$ & $\begin{array}{l}\text { Age } \\
\& \\
\text { Sex }\end{array}$ & $\begin{array}{l}\text { ळ } \\
\text { ڤึ }\end{array}$ & 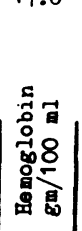 & $\begin{array}{l}\operatorname{ucv} \\
u^{3}\end{array}$ & $\begin{array}{c}\text { MCB } \\
\mu \mu g\end{array}$ & $\begin{array}{c}\text { MCHC } \\
\%\end{array}$ & 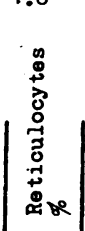 & 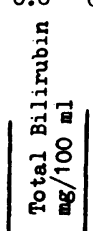 & 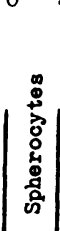 & $\begin{array}{l}\text { OF } \\
1 \%\end{array}$ & $\begin{array}{l}\text { OF } \\
50 \%\end{array}$ & MF & ${ }_{\%}^{\mathrm{AH}}$ & $\underset{\%}{\mathrm{AB}+\mathrm{G}}$ & $\underset{\%}{A B+A d}$ & $\begin{array}{l}\text { Computer } \\
\text { Diagnosis } \\
\text { DC | D } 4\end{array}$ & 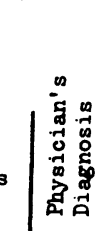 \\
\hline $\begin{array}{r}183-202 \\
203 \\
204 \\
184-201 \\
302 \\
\end{array}$ & $\begin{array}{l}33 F \\
42 F \\
36 F \\
5114 \\
14 F\end{array}$ & $\begin{array}{l}0 \\
0 \\
0 \\
0 \\
0 \\
\end{array}$ & $\begin{array}{l}15.0 \\
13.2 \\
13.3 \\
15.8 \\
13.2 \\
\end{array}$ & $\begin{array}{l}82 \\
84 \\
76 \\
\\
\end{array}$ & $\begin{array}{l}27 \\
28 \\
25 \\
- \\
\end{array}$ & $\begin{array}{l}33 \\
33 \\
32 \\
39 \\
34 \\
\end{array}$ & $\begin{array}{l}1.0 \\
1.4 \\
1.2 \\
1.1 \\
1.5 \\
\end{array}$ & $\begin{array}{l}0.3 \\
0.1 \\
0.4 \\
0.1 \\
0.1\end{array}$ & $\begin{array}{l}0 \\
0 \\
0 \\
0 \\
0 \\
0\end{array}$ & $\begin{array}{r}.45 \\
- \\
- \\
-\end{array}$ & $\begin{array}{r}.38 \\
= \\
=\end{array}$ & $\begin{array}{l}3.8 \\
2.0 \\
5.3 \\
4.7 \\
3.4 \\
\end{array}$ & $\begin{array}{l}2.1 \\
= \\
=\end{array}$ & $\begin{array}{l}0.5 \\
= \\
=\end{array}$ & $\begin{array}{l}0.8 \\
= \\
=\end{array}$ & $\begin{array}{l}\bar{z} \\
\bar{z}\end{array}$ & $\begin{array}{l}- \\
\overline{-} \\
-\end{array}$ \\
\hline $\begin{array}{r}-303 \\
188-201 \\
202 \\
302 \\
303\end{array}$ & $\begin{array}{l}29 M \\
23 F \\
25 M \\
3 F \\
1 M\end{array}$ & $\begin{array}{l}0 \\
- \\
- \\
-\end{array}$ & $\begin{array}{l}15.2 \\
13.9 \\
16.0 \\
11.8 \\
11.3\end{array}$ & 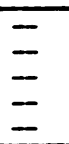 & $\bar{z}$ & $\begin{array}{l}37 \\
=\end{array}$ & $\begin{array}{l}0.3 \\
0.6 \\
0.5 \\
0.4 \\
0.8\end{array}$ & $\begin{array}{l}1.0 \\
\frac{-}{0.0} \\
0.0\end{array}$ & $\begin{array}{l}1 \\
1 \\
0 \\
1 \\
1\end{array}$ & $\begin{array}{r}- \\
.4 \overline{6} \\
\overline{-}\end{array}$ & - & $\begin{array}{l}5.2 \\
1.8 \\
4.8 \\
0.8\end{array}$ & $\bar{z}$ & $\begin{array}{l}\overline{ } \\
\overline{ }\end{array}$ & $\begin{array}{l}\bar{z} \\
\bar{z}\end{array}$ & - & - \\
\hline $\begin{array}{r}189-201 \\
202 \\
302 \\
190-201 \\
202\end{array}$ & $\begin{array}{l}57 X \\
53 F \\
26 F \\
36 X \\
35 F\end{array}$ & $\begin{array}{l}- \\
\overline{-} \\
\overline{0} \\
0\end{array}$ & $\begin{array}{l}15.5 \\
13.9 \\
14.0 \\
16.4 \\
14.3\end{array}$ & $\begin{array}{l}\frac{95}{5} \\
=\end{array}$ & $\begin{array}{l}32 \\
\bar{z}\end{array}$ & $\frac{34}{33}$ & $\begin{array}{l}2.8 \\
4.2 \\
2.9 \\
0.5 \\
1.1\end{array}$ & $\begin{array}{l}\overline{-} \\
\overline{0} \\
0.2 \\
0.7\end{array}$ & $\begin{array}{l}0 \\
2 \\
1 \\
0 \\
3\end{array}$ & $\begin{array}{r}.49 \\
- \\
- \\
-\end{array}$ & $\begin{array}{r}.44 \\
- \\
-\end{array}$ & $\begin{array}{l}7.5 \\
3.5 \\
3.5 \\
4.1 \\
6.5\end{array}$ & $\begin{array}{l}1.7 \\
= \\
=\end{array}$ & $\begin{array}{l}\bar{E} \\
\bar{E}\end{array}$ & $\begin{array}{l}\bar{z} \\
\bar{z}\end{array}$ & - & - \\
\hline $\begin{array}{l}303 \\
304\end{array}$ & $\begin{array}{l}14 X \\
15 F\end{array}$ & $\begin{array}{l}0 \\
0\end{array}$ & $\begin{array}{l}14.1 \\
15.6\end{array}$ & $\bar{z}$ & $\overline{-}$ & $\begin{array}{l}33 \\
33\end{array}$ & $\begin{array}{l}1.2 \\
1.6\end{array}$ & $\begin{array}{l}0.2 \\
0.4\end{array}$ & $\begin{array}{l}0 \\
0\end{array}$ & $\overline{-}$ & $\overline{-}$ & $\begin{array}{l}5.7 \\
6.5\end{array}$ & $\overline{-}$ & $\bar{z}$ & $\overline{-}$ & $\overline{-}$ & $\overline{-}$ \\
\hline Mean & & & 13.8 & 86 & 28.0 & 33 & 2.0 & 0.4 & & .47 & .41 & 4.8 & 4.3 & 0.5 & 2.0 & & \\
\hline Standard $D$ & eviati & & 1.5 & 7.0 & 2.5 & 1.2 & 1.0 & 0.3 & & .05 & .03 & 2.0 & 3.5 & 0.3 & 2.5 & & \\
\hline
\end{tabular}

* Spleen 2 indicates palpable spleen. Spleen $0=$ not felt or not examined; $+=$ affected; $-=$ normal; blank space indicates insufficient data to form discriminant. See Table I for other abbreviations. 
son (17). Mechanical fragility was done by modification of the method of Shen, Castle and Fleming (18) and osmotic fragility by the method of Emerson and co-workers (19). Interpolated values of saline concentration giving 1 and 50 per cent hemolysis were recorded for numerical analysis. Autohemolysis was done by the method of Young, Izzo, Altman and Swisher (20). To some aliquots glucose was added to give a final concentration of $2.7 \times 10^{-2} \mathrm{M}$, or adenosine was added to give a final concentration of $9 \times 10^{-3} \mathrm{M}$.

5. Discriminatory analysis. It is known that many laboratory tests related to hemolysis are abnormal in hereditary spherocytosis. Statistical theory indicates that a combination of these tests would provide a better diagnostic measure than would any isolated test. This statement should be true until the genotype can be identified. A suitable statistical tool for using multiple laboratory tests was developed by Fisher $(21,22)$. This is the discriminant function method; it gives a formula for the best linear combination of measurements for distinguishing between groups and is analogous to multiple regression. The formula gives an optimal discriminant only if the distributions are normal and have the same standard deviations in the two groups. Although these conditions are only approximately fulfilled, the method has been found in practice to give good results.

Twenty-nine affected cases with intact spleens were selected as classical examples of $\mathrm{HS}$ on the basis of indisputable laboratory evidence and family history. Values for hemoglobin, erythrocyte indices, reticulocyte percentage, total bilirubin, spherocyte score, 1 and 50 per cent osmotic fragility, mechanical fragility, autohemolysis, autohemolysis with glucose, and autohemolysis with adenosine from these 29 typical cases were compared with similar data from 48 controls. The data were processed by an IBM-650 computer programmed to maximize the intraclass correlations of the two groups. A derived number was obtained for each subject, representing the weighted value of the combination of all 13 tests. This derived number was called the complete discriminant score (Dc). Zero was chosen as the dividing line between normal (negative score) and affected subjects (positive score). As might have been anticipated, there was no overlap of Dc values when the control group was compared with the classical cases of HS. A second score, using only the values for reticulocytes, bili-

TABLE III

Laboratory data of 76 affected family members with spleens intact*

\begin{tabular}{|c|c|c|c|c|c|c|c|c|c|c|c|c|c|c|c|c|c|c|}
\hline $\begin{array}{c}\text { Family and } \\
\text { Individual } \\
\text { Number }\end{array}$ & $\begin{array}{l}\text { Age } \\
\& \\
\text { Sex }\end{array}$ & 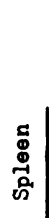 & 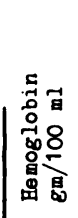 & $\begin{array}{l}\mathrm{MCV} \\
\mathrm{u}^{3}\end{array}$ & $\begin{array}{r}\text { MCH } \\
\mu \mu g\end{array}$ & $\begin{array}{c}\text { MCHC } \\
\%\end{array}$ & 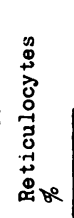 & 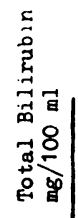 & 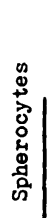 & $\begin{array}{l}O F \\
1 \%\end{array}$ & $\begin{array}{l}\text { OF } \\
50 \%\end{array}$ & $\begin{array}{l}\text { MF } \\
\%\end{array}$ & ${ }_{\%}^{\mathrm{AH}}$ & $\underset{\%}{\mathrm{AB}+\mathrm{C}}$ & $\underset{\%}{\mathrm{AH}+\mathrm{Ad}}$ & $\begin{array}{l}\text { Compu } \\
\text { Diagn } \\
\text { Dc }\end{array}$ & $\begin{array}{l}\text { ter } \\
\text { osis } \\
D_{4}\end{array}$ & 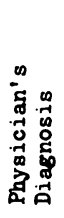 \\
\hline $\begin{array}{r}111-201 \\
112-201 \\
166-201 \\
301 \\
167-301 \\
\end{array}$ & $\begin{array}{l}23 M \\
11 F \\
58 M \\
23 M \\
10 X \\
\end{array}$ & $\begin{array}{l}2 \\
2 \\
2 \\
2 \\
2 \\
\end{array}$ & $\begin{array}{r}15.8 \\
8.0 \\
8.9 \\
13.4 \\
11.0 \\
\end{array}$ & $\begin{array}{l}66 \\
88 \\
84 \\
96 \\
72 \\
\end{array}$ & $\begin{array}{l}23 \\
24 \\
30 \\
35 \\
24 \\
\end{array}$ & $\begin{array}{l}35 \\
28 \\
36 \\
33 \\
34 \\
\end{array}$ & $\begin{array}{r}15.6 \\
31.9 \\
14.9 \\
8.9 \\
15.1 \\
\end{array}$ & $\begin{array}{l}4.1 \\
1.5 \\
2.7 \\
4.4 \\
0.5 \\
\end{array}$ & $\begin{array}{l}2 \\
3 \\
2 \\
2 \\
2 \\
\end{array}$ & $\begin{array}{l}.44 \\
.49 \\
.60 \\
.48 \\
.64 \\
\end{array}$ & $\begin{array}{l}.39 \\
.44 \\
.43 \\
.42 \\
.44 \\
\end{array}$ & $\begin{array}{l}14.0 \\
15.7 \\
21.8 \\
14.9 \\
17.8 \\
\end{array}$ & $\begin{array}{r}3.0 \\
14.9 \\
14.8 \\
39.6 \\
48.5 \\
\end{array}$ & $\begin{array}{r}0.3 \\
10.6 \\
2.2 \\
1.4 \\
6.6 \\
\end{array}$ & $\begin{array}{r}2.7 \\
24.4 \\
9.0 \\
17.0 \\
\end{array}$ & $\begin{array}{l}+ \\
+ \\
+ \\
+ \\
+\end{array}$ & $\begin{array}{l}+ \\
+ \\
+ \\
+ \\
+\end{array}$ & $\begin{array}{l}+ \\
+ \\
+ \\
+ \\
+\end{array}$ \\
\hline $\begin{array}{r}303 \\
304 \\
168-202 \\
205 \\
401 \\
\end{array}$ & $\begin{array}{r}8 F \\
2 F \\
74 M \\
65 F \\
7 F \\
\end{array}$ & $\begin{array}{l}0 \\
0 \\
2 \\
2 \\
0 \\
\end{array}$ & $\begin{array}{l}11.5 \\
12.0 \\
16.6 \\
14.5 \\
13.6 \\
\end{array}$ & $\frac{-}{107} \frac{-}{72}$ & $\begin{array}{l}26 \\
27 \\
38 \\
27 \\
\end{array}$ & $\begin{array}{l}- \\
- \\
33 \\
38 \\
37\end{array}$ & $\begin{array}{r}12.1 \\
10.3 \\
3.0 \\
7.6 \\
6.1 \\
\end{array}$ & $\begin{array}{l}1.1 \\
0.7 \\
1.2 \\
0.7\end{array}$ & $\begin{array}{l}3 \\
3 \\
1 \\
3 \\
2 \\
\end{array}$ & $\frac{.}{.49}$ & $\frac{-}{.43}$ & $\begin{array}{r}12.7 \\
8.2 \\
15.9 \\
10.2 \\
\end{array}$ & $\begin{array}{l}- \\
\overline{23.2} \\
\overline{4.8}\end{array}$ & $\begin{array}{l}\overline{-} \\
\overline{1.4} \\
\bar{z}\end{array}$ & $\begin{array}{l}- \\
7.0 \\
-\end{array}$ & - & $\begin{array}{l}- \\
+ \\
+\end{array}$ & $\begin{array}{l}+ \\
+ \\
+ \\
+\end{array}$ \\
\hline $\begin{array}{r}402 \\
403 \\
169-201 \\
301 \\
302 \\
\end{array}$ & $\begin{array}{r}8 F \\
4 F \\
68 F \\
21 F \\
24 F \\
\end{array}$ & $\begin{array}{l}0 \\
2 \\
0 \\
2 \\
2 \\
\end{array}$ & $\begin{array}{r}14.8 \\
9.1 \\
12.3 \\
13.3 \\
13.1 \\
\end{array}$ & $\begin{array}{l}- \\
\overline{83} \\
86 \\
77\end{array}$ & $\begin{array}{l}- \\
\overline{27} \\
31 \\
26\end{array}$ & $\begin{array}{l}\frac{38}{33} \\
36 \\
34 \\
\end{array}$ & $\begin{array}{r}3.1 \\
12.4 \\
13.0 \\
9.8 \\
10.8 \\
\end{array}$ & $\begin{array}{l}0.9 \\
0.5 \\
1.6 \\
2.0 \\
1.1 \\
\end{array}$ & $\begin{array}{l}3 \\
3 \\
2 \\
2 \\
2 \\
\end{array}$ & $\begin{array}{r}- \\
.50 \\
.53 \\
.47 \\
\end{array}$ & $\begin{array}{l}- \\
. \\
.44 \\
.43 \\
.42 \\
\end{array}$ & $\begin{array}{r}15.6 \\
8.8 \\
12.8 \\
9.0 \\
\end{array}$ & $\begin{array}{c}- \\
- \\
5.1 \\
30.7 \\
- \\
\end{array}$ & $\begin{array}{l}- \\
- \\
3.3 \\
2.3 \\
1.0\end{array}$ & $\begin{array}{r}- \\
\overline{5.7} \\
16.0 \\
5.7 \\
\end{array}$ & + & $\begin{array}{l}+ \\
+ \\
+ \\
+ \\
+\end{array}$ & $\begin{array}{l}+ \\
+ \\
+ \\
+ \\
+\end{array}$ \\
\hline $\begin{array}{r}303 \\
304 \\
306 \\
309 \\
407 \\
\end{array}$ & $\begin{array}{l}24 F \\
31 F \\
36 M \\
38 F \\
11 M \\
\end{array}$ & $\begin{array}{l}2 \\
2 \\
2 \\
2 \\
2 \\
\end{array}$ & $\begin{array}{l}12.1 \\
13.8 \\
14.4 \\
13.8 \\
12.3 \\
\end{array}$ & $\begin{array}{l}90 \\
-5 \\
84 \\
84 \\
-\end{array}$ & $\begin{array}{l}\frac{32}{30} \\
29 \\
-- \\
\end{array}$ & $\begin{array}{l}36 \\
36 \\
36 \\
35 \\
37 \\
\end{array}$ & $\begin{array}{r}10.4 \\
3.4 \\
13.5 \\
8.7 \\
5.2 \\
\end{array}$ & $\begin{array}{l}2.5 \\
1.0 \\
1.1 \\
1.0 \\
1.6 \\
\end{array}$ & $\begin{array}{l}3 \\
3 \\
3 \\
3 \\
3 \\
\end{array}$ & $\begin{array}{l}.50 \\
.47 \\
.52 \\
-\end{array}$ & $\begin{array}{r}.44 \\
.42 \\
.46 \\
-\end{array}$ & $\begin{array}{r}10.4 \\
9.5 \\
13.2 \\
11.8 \\
13.6 \\
\end{array}$ & $\begin{array}{c}20.3 \\
- \\
22.3 \\
7.5 \\
- \\
\end{array}$ & $\begin{array}{l}1.6 \\
- \\
1.3 \\
2.1 \\
-\end{array}$ & $\begin{array}{l}\frac{6.7}{-} \\
3.6 \\
\end{array}$ & $\begin{array}{l}+ \\
+ \\
+\end{array}$ & $\begin{array}{l}+ \\
+ \\
+ \\
+ \\
+\end{array}$ & $\begin{array}{l}+ \\
+ \\
+ \\
+ \\
+\end{array}$ \\
\hline $\begin{array}{r}408 \\
170-301 \\
303 \\
304 \\
407 \\
\end{array}$ & $\begin{array}{l}14 M \\
42 M \\
48 M \\
48 M \\
18 \mathrm{~F} \\
\end{array}$ & $\begin{array}{l}2 \\
0 \\
2 \\
2 \\
2 \\
\end{array}$ & $\begin{array}{l}15.4 \\
17.1 \\
11.9 \\
13.9 \\
14.1\end{array}$ & $\begin{array}{l}- \\
98 \\
92 \\
84 \\
86\end{array}$ & $\begin{array}{l}\overline{36} \\
31 \\
30 \\
31\end{array}$ & $\begin{array}{l}38 \\
38 \\
34 \\
35 \\
35\end{array}$ & $\begin{array}{r}3.8 \\
8.0 \\
12.8 \\
8.5 \\
4.8\end{array}$ & $\begin{array}{l}1.1 \\
1.7 \\
2.6 \\
1.8 \\
0.7\end{array}$ & $\begin{array}{l}3 \\
3 \\
2 \\
3 \\
1\end{array}$ & $\begin{array}{l}. \\
.55 \\
.46 \\
.49 \\
.49\end{array}$ & $\begin{array}{l}-\overline{.44} \\
.44 \\
.44 \\
.43 \\
\end{array}$ & $\begin{array}{r}9.4 \\
10.7 \\
17.1 \\
13.1 \\
15.1 \\
\end{array}$ & $\begin{array}{c}-\overline{30.2} \\
26.1 \\
24.1 \\
29.2\end{array}$ & $\begin{array}{l}-1 \\
2.5 \\
1.6 \\
0.9 \\
1.4 \\
\end{array}$ & $\begin{array}{r}- \\
9.5 \\
7.9 \\
3.9 \\
10.6 \\
\end{array}$ & $\begin{array}{l}+ \\
+ \\
+ \\
+\end{array}$ & $\begin{array}{l}+ \\
+ \\
+ \\
+ \\
-\end{array}$ & $\begin{array}{l}+ \\
+ \\
+ \\
+ \\
+\end{array}$ \\
\hline $\begin{array}{r}409 \\
416 \\
171-301 \\
172-401 \\
402 \\
\end{array}$ & $\begin{array}{l}16 F \\
19 F \\
35 M \\
19 M \\
18 M \\
\end{array}$ & $\begin{array}{l}0 \\
2 \\
0 \\
0 \\
2 \\
\end{array}$ & $\begin{array}{l}15.0 \\
13.6 \\
16.1 \\
11.9 \\
12.9 \\
\end{array}$ & $\begin{array}{l}85 \\
85 \\
83 \\
- \\
- \\
\end{array}$ & $\begin{array}{l}31 \\
31 \\
29 \\
- \\
--\end{array}$ & $\begin{array}{l}36 \\
36 \\
36 \\
33 \\
37 \\
\end{array}$ & $\begin{array}{r}5.6 \\
6.6 \\
6.5 \\
8.1 \\
12.5 \\
\end{array}$ & $\begin{array}{l}0.8 \\
1.1 \\
1.0 \\
2.4 \\
4.7 \\
\end{array}$ & $\begin{array}{l}2 \\
3 \\
3 \\
3 \\
3 \\
\end{array}$ & $\begin{array}{l}.46 \\
.50 \\
.53 \\
.62 \\
.68 \\
\end{array}$ & $\begin{array}{l}.43 \\
.42 \\
.46 \\
.46 \\
.45 \\
\end{array}$ & $\begin{array}{r}9.8 \\
12.8 \\
9.7 \\
24.4 \\
16.0 \\
\end{array}$ & $\begin{array}{r}15.3 \\
21.3 \\
9.8 \\
- \\
- \\
\end{array}$ & $\begin{array}{l}0.2 \\
0.9 \\
0.7 \\
- \\
-\end{array}$ & $\begin{array}{l}4.0 \\
7.5 \\
2.6 \\
-- \\
-- \\
\end{array}$ & $\begin{array}{l}+ \\
+ \\
+\end{array}$ & $\begin{array}{l}+ \\
+ \\
+ \\
+ \\
+\end{array}$ & $\begin{array}{l}+ \\
+ \\
+ \\
+ \\
+\end{array}$ \\
\hline $\begin{array}{r}405 \\
408 \\
173-205 \\
301 \\
304\end{array}$ & $\begin{array}{r}13 M \\
3 M \\
63 F \\
43 M \\
35 M\end{array}$ & $\begin{array}{l}2 \\
0 \\
0 \\
0 \\
2\end{array}$ & $\begin{array}{l}14.8 \\
11.5 \\
10.6 \\
12.6 \\
13.2\end{array}$ & $\begin{array}{l}- \\
85 \\
85 \\
94\end{array}$ & $\begin{array}{l}- \\
\overline{28} \\
29 \\
34\end{array}$ & $\begin{array}{l}39 \\
38 \\
33 \\
35 \\
36\end{array}$ & $\begin{array}{r}13.0 \\
12.9 \\
7.0 \\
9.3 \\
6.0\end{array}$ & $\begin{array}{l}1.7 \\
0.6 \\
1.5 \\
0.8 \\
1.8\end{array}$ & $\begin{array}{l}3 \\
3 \\
3 \\
2 \\
3\end{array}$ & $\begin{array}{l}.67 \\
.62 \\
.49 \\
.53 \\
.53\end{array}$ & $\begin{array}{l}.43 \\
.45 \\
.45 \\
.44 \\
.45\end{array}$ & $\begin{array}{r}15.2 \\
17.0 \\
7.8 \\
17.3 \\
12.4\end{array}$ & $\begin{array}{c}26.9 \\
- \\
33.1 \\
30.8 \\
-\end{array}$ & $\begin{array}{l}5.5 \\
1.0 \\
2.1 \\
-\end{array}$ & $\begin{array}{l}9.6 \\
\overline{9.2} \\
5.5 \\
-\end{array}$ & + & $\begin{array}{l}+ \\
+ \\
+ \\
+ \\
+\end{array}$ & $\begin{array}{l}+ \\
+ \\
+ \\
+ \\
+\end{array}$ \\
\hline
\end{tabular}




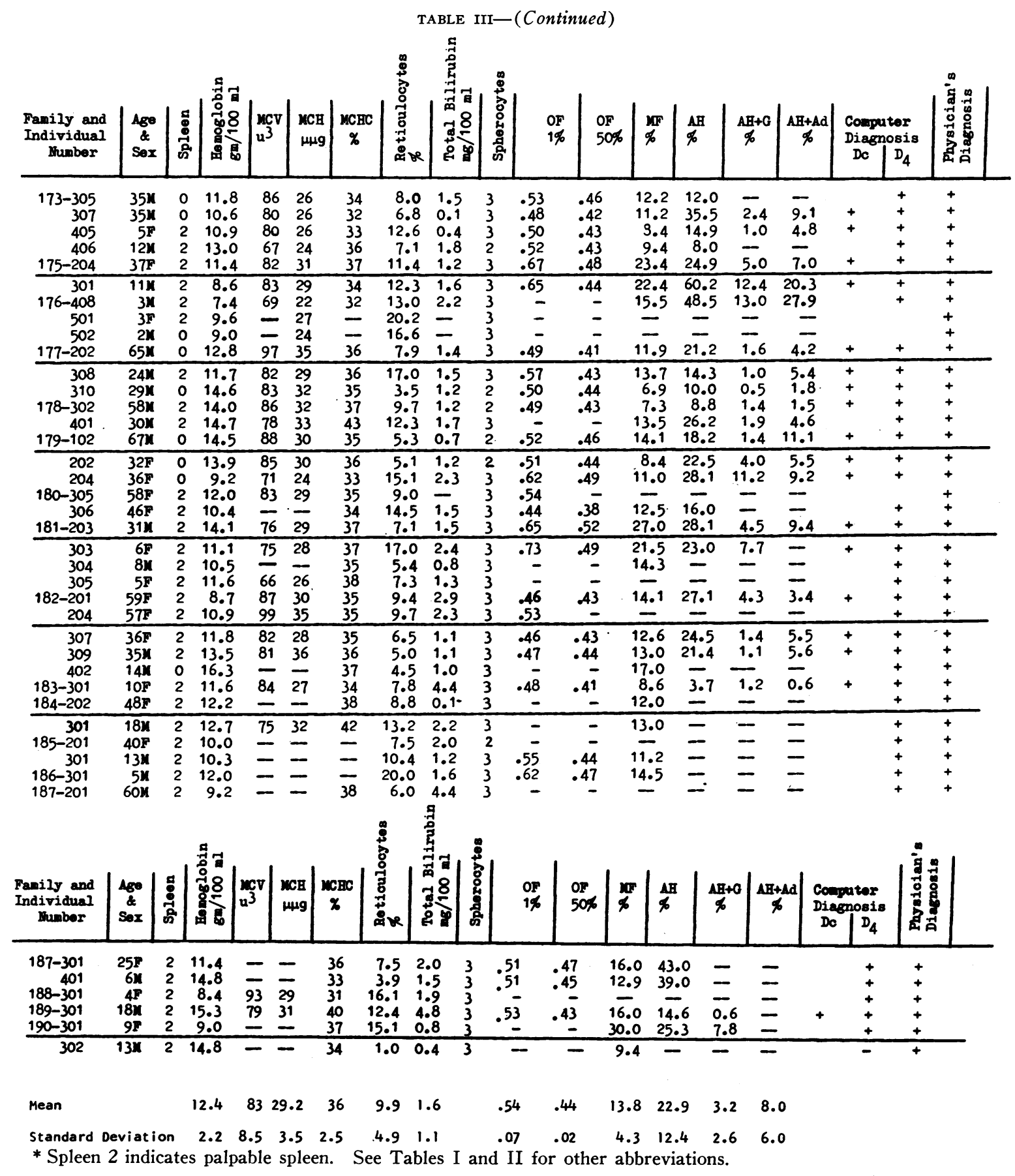

rubin, hemoglobin, and spherocytes, hereafter called $D_{4}$, was similarly prepared. A third score, called $D_{5}$, was made, adding mechanical fragility to the data for $D_{4}$. A fourth discriminant, called $\mathrm{Dh}$, using autohemolysis, autohemolysis with glucose, osmotic fragility 1 and 50 per cent, and mechanical fragility was also made. These four discriminants were used to determine relative effectiveness of various combinations of tests.
On the basis of the variables weighted by analysis of normals and classical cases, the computer then scored the remaining family members as "unknowns," deriving for each family member a $D_{4}$ and Dc. A physician's diagnosis was made by one of us (RFS), who examined the same data available to the computer for Dc; i.e., with names and computer scores excluded to minimize bias. In his analysis of the data the physician took into 
TABLE IV

Laboratory data of 19 affected family members zeith spleens removed *

\begin{tabular}{|c|c|c|c|c|c|c|c|c|c|c|c|c|c|c|c|c|c|}
\hline $\begin{array}{l}\text { Family and } \\
\text { Individual } \\
\text { Number }\end{array}$ & $\begin{array}{l}\text { Age } \\
\& \\
\text { Sex }\end{array}$ & 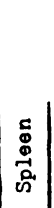 & 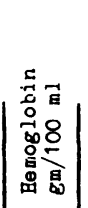 & $\begin{array}{l}m c v \\
u^{3}\end{array}$ & $\begin{array}{c}\text { MCH } \\
\mu \mu \mathrm{g}\end{array}$ & $\begin{array}{c}\text { MCHC } \\
\%\end{array}$ & 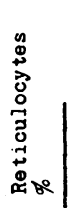 & 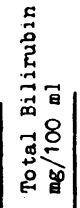 & 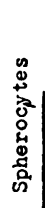 & $\begin{array}{l}\text { OF } \\
1 \%\end{array}$ & $\begin{array}{l}\text { OF } \\
50 \%\end{array}$ & $\begin{array}{l}\mathrm{MF} \\
\%\end{array}$ & $\begin{array}{l}\mathrm{AH} \\
\%\end{array}$ & $\underset{\%}{\mathrm{AH}+\mathrm{C}}$ & $\underset{\%}{\mathrm{AH}+\mathrm{Ad}}$ & $\begin{array}{l}\text { Computer } \\
\text { Diagnosis } \\
\text { Dc } \mid D_{4}\end{array}$ & 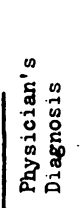 \\
\hline $\begin{array}{r}167-202 \\
168-301 \\
169-307 \\
308 \\
170-305 \\
\end{array}$ & $\begin{array}{l}40 M \\
42 M \\
30 M \\
32 M \\
46 \mathrm{~F} \\
\end{array}$ & $\begin{array}{l}1 \\
1 \\
1 \\
1 \\
1 \\
\end{array}$ & $\begin{array}{l}15.9 \\
16.5 \\
16.2 \\
15.9 \\
15.8 \\
\end{array}$ & $\begin{array}{l}77 \\
77 \\
88 \\
95 \\
91 \\
\end{array}$ & $\begin{array}{l}26 \\
27 \\
30 \\
34 \\
33 \\
\end{array}$ & $\begin{array}{l}34 \\
38 \\
34 \\
36 \\
37 \\
\end{array}$ & $\begin{array}{l}3.9 \\
2.4 \\
3.8 \\
3.0 \\
\end{array}$ & $\begin{array}{l}0.5 \\
1.0 \\
0.8 \\
0.9 \\
\end{array}$ & $\begin{array}{l}2 \\
3 \\
1 \\
1 \\
1 \\
\end{array}$ & $\begin{array}{l}.58 \\
.51 \\
.51 \\
.51 \\
.54 \\
\end{array}$ & $\begin{array}{l}.49 \\
.42 \\
.45 \\
.43 \\
.44\end{array}$ & $\begin{array}{r}15.6 \\
12.0 \\
9.5 \\
7.0 \\
8.4 \\
\end{array}$ & $\begin{array}{r}42.4 \\
7.1 \\
38.6 \\
21.3 \\
23.1 \\
\end{array}$ & $\begin{array}{l}0.8 \\
0.4 \\
1.5 \\
1.0 \\
0.9\end{array}$ & $\begin{array}{l}26.9 \\
9.0 \\
8.6 \\
6.7\end{array}$ & $\begin{array}{l}+ \\
- \\
-\end{array}$ & $\begin{array}{l}+ \\
+ \\
+ \\
+ \\
+\end{array}$ \\
\hline $\begin{array}{r}306 \\
171-304 \\
172-403 \\
406 \\
407\end{array}$ & $\begin{array}{l}44 F \\
43 F \\
20 M \\
17 M \\
13 M\end{array}$ & $\begin{array}{l}1 \\
1 \\
1 \\
1 \\
1\end{array}$ & $\begin{array}{l}15.0 \\
14.2 \\
16.8 \\
16.6 \\
14.6\end{array}$ & $\begin{array}{l}93 \\
82 \\
92 \\
74 \\
79\end{array}$ & $\begin{array}{l}33 \\
26 \\
33 \\
27 \\
27\end{array}$ & $\begin{array}{l}35 \\
31 \\
36 \\
37 \\
32 \\
\end{array}$ & $\begin{array}{l}-\overline{2.9} \\
3.9 \\
4.7 \\
8.4\end{array}$ & $\begin{array}{l}0.5 \\
0.8 \\
0.5 \\
0.4 \\
0.3\end{array}$ & $\begin{array}{l}2 \\
3 \\
3 \\
1 \\
2 \\
\end{array}$ & $\begin{array}{l}.54 \\
.52 \\
.58 \\
.55 \\
.53\end{array}$ & $\begin{array}{l}.45 \\
.45 \\
.47 \\
.46 \\
.45\end{array}$ & $\begin{array}{r}10.4 \\
6.8 \\
17.0 \\
15.1 \\
13.3\end{array}$ & $\begin{array}{l}22.9 \\
13.2 \\
93.5 \\
82.0 \\
10.4\end{array}$ & $\begin{array}{l}0.7 \\
1.1 \\
7.8 \\
4.0 \\
0.5\end{array}$ & $\begin{array}{r}8.4 \\
5.3 \\
44.2 \\
26.0 \\
8.0\end{array}$ & $\begin{array}{l}+ \\
+ \\
+ \\
+ \\
+\end{array}$ & $\begin{array}{l}+ \\
+ \\
+ \\
+ \\
+ \\
\end{array}$ \\
\hline $\begin{array}{r}411 \\
174-302 \\
176-302 \\
303 \\
406 \\
\end{array}$ & $\begin{array}{l}28 \mathrm{~F} \\
37 M \\
44 M \\
42 M \\
8 M \\
\end{array}$ & $\begin{array}{l}1 \\
1 \\
1 \\
1 \\
1 \\
\end{array}$ & $\begin{array}{l}15.6 \\
14.3 \\
14.9 \\
14.7 \\
13.3 \\
\end{array}$ & $\begin{array}{l}82 \\
81 \\
93 \\
76 \\
81 \\
\end{array}$ & $\begin{array}{l}28 \\
27 \\
32 \\
25 \\
30 \\
\end{array}$ & $\begin{array}{l}35 \\
34 \\
34 \\
33 \\
37 \\
\end{array}$ & $\begin{array}{l}1.3 \\
4.0 \\
6.5 \\
2.8 \\
5.3 \\
\end{array}$ & $\begin{array}{l}0.6 \\
0.2 \\
0.5 \\
0.5 \\
0.4 \\
\end{array}$ & $\begin{array}{l}2 \\
3 \\
3 \\
3 \\
2 \\
\end{array}$ & $\begin{array}{l}.58 \\
.56 \\
.55 \\
.55 \\
.57 \\
\end{array}$ & $\begin{array}{l}.48 \\
.48 \\
.46 \\
.47 \\
.46\end{array}$ & $\begin{array}{l}10.8 \\
12.5 \\
12.0 \\
16.2 \\
21.9 \\
\end{array}$ & $\begin{array}{l}35.0 \\
35: 8 \\
35.5 \\
50.8 \\
32.7\end{array}$ & $\begin{array}{l}0.8 \\
1.2 \\
0.7 \\
8.1 \\
0.9\end{array}$ & $\begin{array}{r}13.9 \\
17.7 \\
26.8 \\
22.8 \\
6.9 \\
\end{array}$ & $\begin{array}{l}- \\
+ \\
+ \\
+ \\
+\end{array}$ & $\begin{array}{l}+ \\
+ \\
+ \\
+ \\
+\end{array}$ \\
\hline $\begin{array}{r}407 \\
423 \\
180-301 \\
186-201\end{array}$ & $\begin{array}{l}7 F \\
27 F \\
51 M \\
31 F\end{array}$ & $\begin{array}{l}1 \\
1 \\
1 \\
1\end{array}$ & $\begin{array}{l}13.4 \\
11.7 \\
15.6 \\
14.5\end{array}$ & $\begin{array}{l}81 \\
77 \\
87 \\
-\end{array}$ & $\begin{array}{l}29 \\
24 \\
31 \\
-\end{array}$ & $\begin{array}{l}35 \\
32 \\
35 \\
-\end{array}$ & $\begin{array}{l}6.7 \\
5.2 \\
3.0 \\
-\end{array}$ & $\begin{array}{l}0.3 \\
0.3 \\
0.2 \\
-\end{array}$ & $\begin{array}{r}3 \\
1 \\
3 \\
-\end{array}$ & $\begin{array}{l}.53 \\
.45 \\
.55 \\
.57\end{array}$ & $\begin{array}{l}.46 \\
.43 \\
.46 \\
.48\end{array}$ & $\begin{array}{r}17.5 \\
17.8 \\
8.5 \\
14.8\end{array}$ & $\begin{array}{l}37.2 \\
15.6 \\
45.7 \\
-\end{array}$ & $\begin{array}{l}0.8 \\
0.5 \\
6.1 \\
-\end{array}$ & $\begin{array}{r}9.2 \\
6.2 \\
30.9 \\
--\end{array}$ & $\begin{array}{l}+ \\
+ \\
+\end{array}$ & $\begin{array}{l}+ \\
+ \\
+\end{array}$ \\
\hline Mean & & & 15.0 & 84 & 29 & 35 & 4.2 & 0.5 & & .54 & .46 & 13.0 & 35.7 & 2.1 & 16.3 & & \\
\hline standard & : & & 1.30 & 6.8 & 3.1 & 1.7 & 1.8 & 0.2 & & .03 & .02 & 4.1 & 22.7 & 2.6 & 11.3 & & \\
\hline
\end{tabular}

account whether or not the spleen was palpable. The machine did not score this item as part of Dc because the control group had not been examined for palpability of the spleen.

\section{RESULTS}

Tables I-IV give data for four groups: I, normal controls; II, nonaffected family members; III, affected relatives with spleen intact; and IV, affected relatives after splenectomy. Table $\mathrm{V}$ gives the means and standard deviations for the several laboratory tests of these four groups.
Multiple correlation coefficients for various combinations of laboratory tests are given in Table VI. These coefficients were determined by comparing the controls with the classical cases of HS. If the distributions were normal and the variances the same for these two groups, and if affected persons were representative of all carriers instead of clearly affected cases, there would be a simple relationship between the correlation coefficients and the overlap. These conditions were not met, and we have no simple method of calculating the amount

TABLE $V$

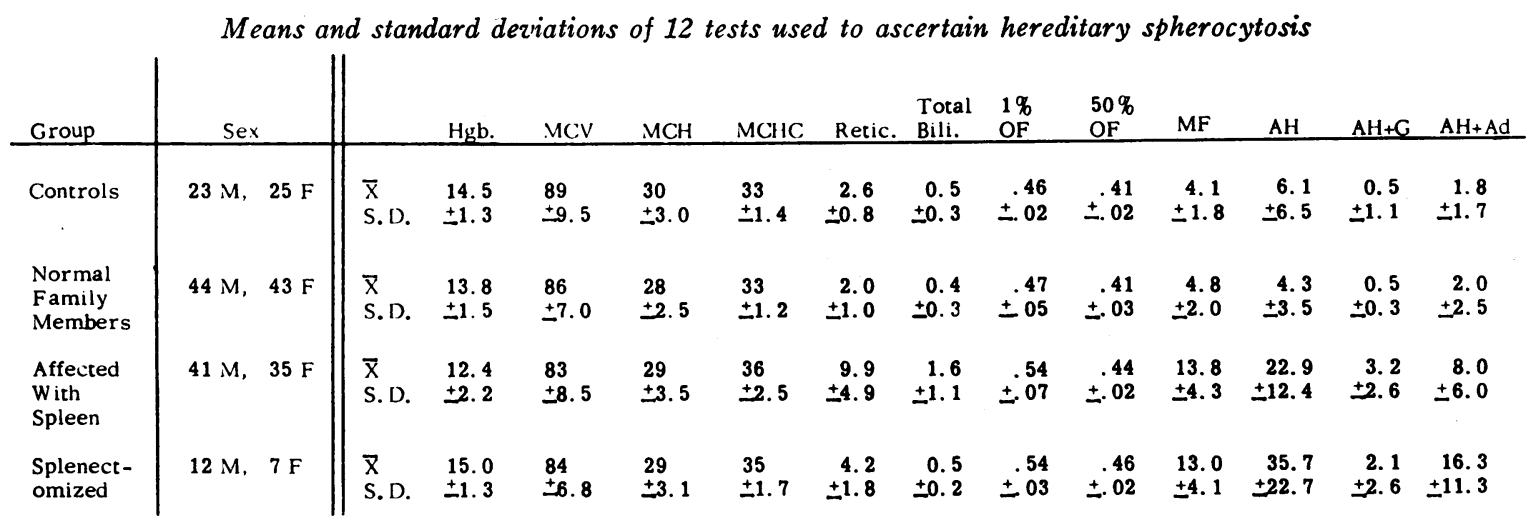


of overlap between normal subjects and carriers of the gene for spherocytosis. Table VII contains correlation coefficients of laboratory tests for normal controls and classical spherocytic patients.

The equation for $D_{4}$ is : $-0.9797+0.1842$ (spherocyte score) -0.0255 (hemoglobin) + 0.0471 (ln total bilirubin $\mathrm{mg}$ per L) +0.2735 ( $\mathrm{ln}$ reticulocytes per 1,000$)$.

A sample solution for the data of Subject 187301 in which the spherocyte score was 3 , the hemoglobin $11.4 \mathrm{~g}$, the total bilirubin $20 \mathrm{mg}$ per $\mathrm{L}$, and the reticulocytes 75 per 1,000 is:

$\mathrm{D}_{4}=-0.9797+0.1842(3.0)-0.0255(11.4)+$ $0.0471(\ln 20)+0.2735(\ln 75)=+0.60$

Since this is a positive score, by the use of this discriminant the patient was considered affected.

The standard deviations of tests performed on the control group are wide. This is largely due to skewing of curves to the right. Day-to-day variability for a given individual was also noted, especially in the autohemolysis test. For example,
TABLE VI

Multiple correlation coefficients of various combinations of laboratory tests used to form discriminants

\begin{tabular}{|c|c|c|}
\hline $\begin{array}{c}\text { Discriminant } \\
\mathrm{D}_{4}\end{array}$ & $\begin{array}{l}\text { Discriminant } \\
\text { Ratios } \times 100 \\
88.1 \%\end{array}$ & $\begin{array}{l}\text { Components used to form discriminant } \\
\text { Hemoglobin, Reticulocytes, Bilirubin, } \\
\text { Spherocytes }\end{array}$ \\
\hline$D_{5}$ & $89.6 \%$ & $\begin{array}{l}\text { Hemoglobin, Reticulocytes, Bilirubin, } \\
\text { Spherocytes, MF }\end{array}$ \\
\hline Dc & $91.4 \%$ & $\begin{array}{l}\text { Hemoglobin, Indices, Reticulocytes, } \\
\text { Bilirubin, Spherocytes, MF, OF } 1 \% \text {, } \\
\text { OF 50\%, AH, AH + G, AH + Ad }\end{array}$ \\
\hline Dh & $78.5 \%$ & $M F$, OF $1 \%$. OF 50\%, AH, AH +G \\
\hline
\end{tabular}

the standard deviation of six replicate autohemolysis determinations with a mean of 18.3 per cent was 0.7. Over a period of several months autohemolysis was determined six times on blood from a single nonfasting individual. The mean value was 7.4 per cent with a standard deviation of 4.9.

Figures 1-4 illustrate graphically the overlap between normal and affected family members when single tests were used. Figure 1 shows the distribution of reticulocyte percentages for affected persons with a spleen and nonaffected family members. It can be seen that the reticulocyte count is

$$
\begin{aligned}
& \text { TABLE VII } \\
& \text { Correlations of single tests used to diagnose hereditary spherocytosis }
\end{aligned}
$$

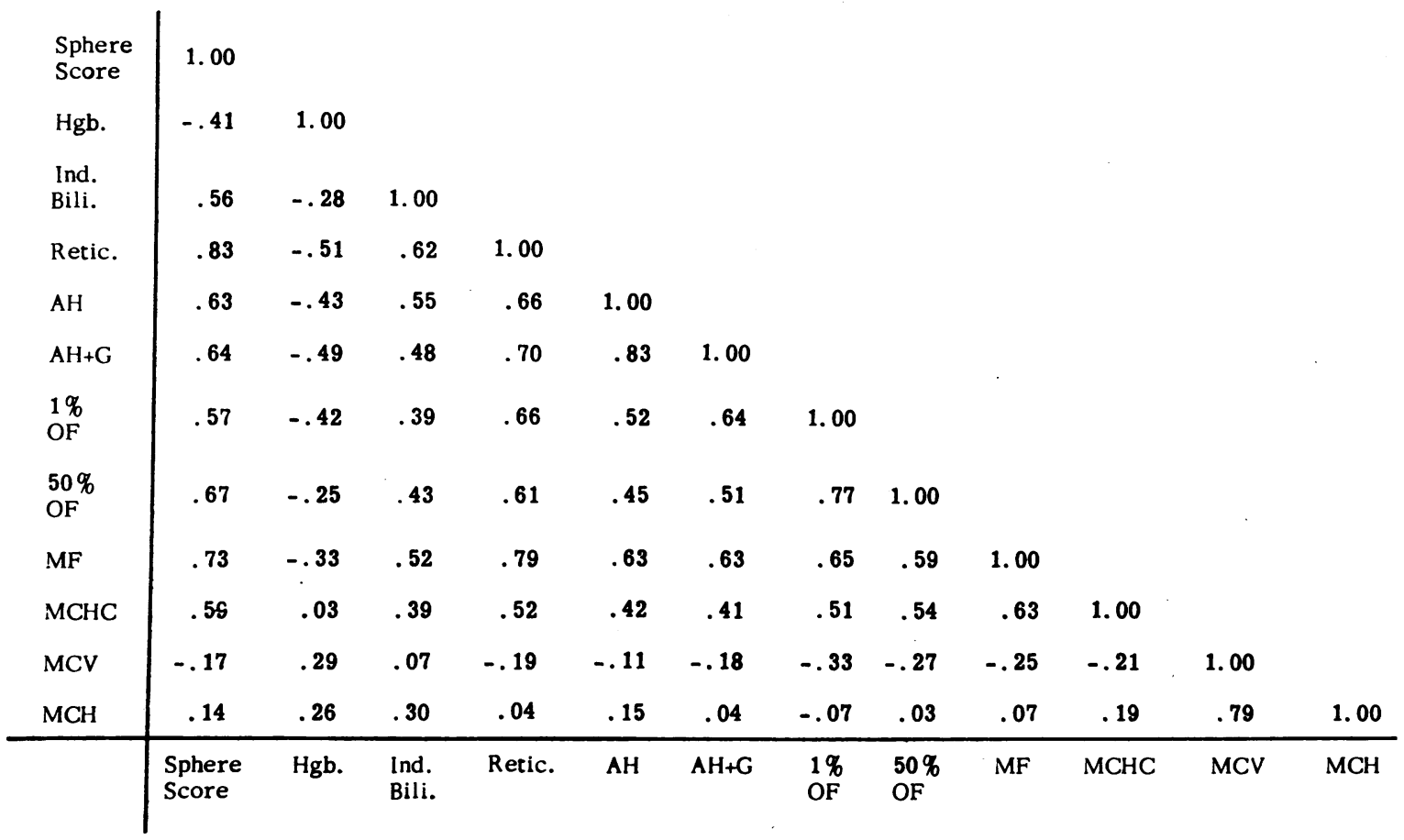

$$
\begin{aligned}
& \text { If } \mathrm{r} \text { (correlation coefficient) }= \pm .22, p=0.05 \\
& \text { If } \mathrm{r} \text { (correlation coefficient) }= \pm .29, p=0.01 \\
& \text { If } \mathrm{r} \text { (correlation coefficient) }= \pm .37, \mathrm{p}=0.001
\end{aligned}
$$




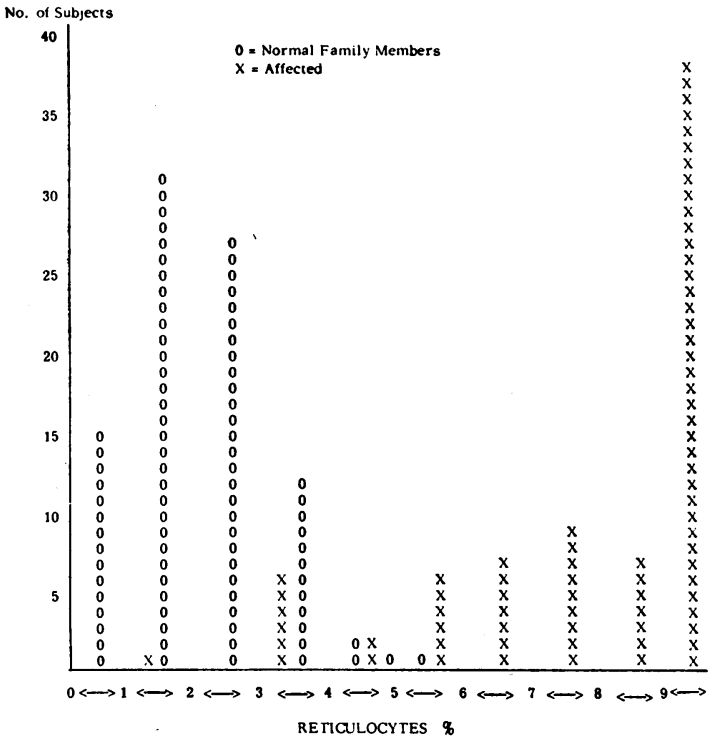

Fig. 1. Distribution of Reticulocyte' percentages FOR AFFECTED AND NORMAL FAMILY MEMBERS WITH SPLEENS INTACT. Each symbol indicates one subject.

No. of Subjects

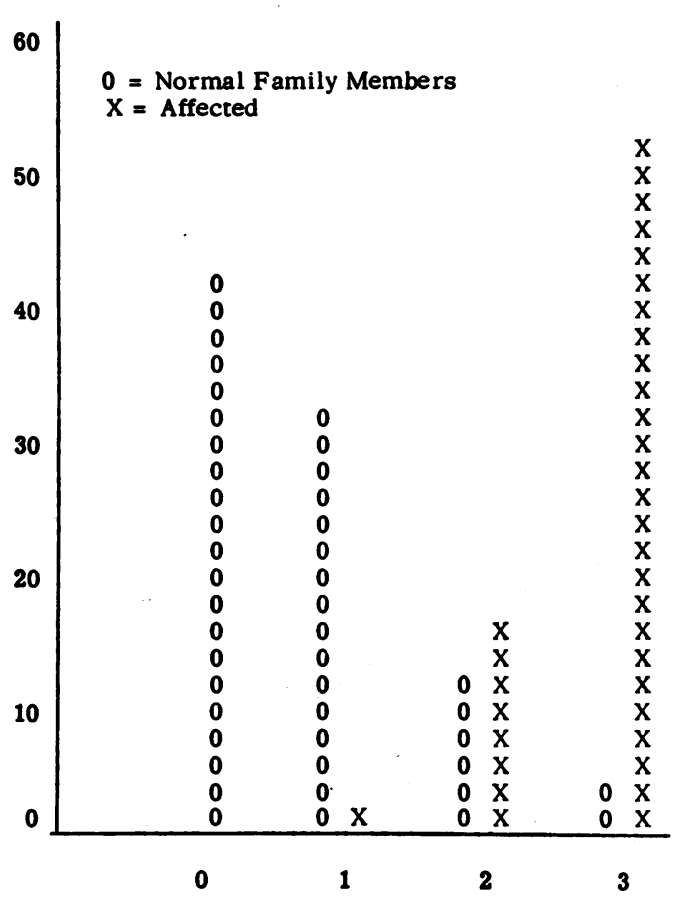

SPHEROCYTE SCORE

Fig. 2. Distribution OF SPHEROCYTE SCORES FOR AFFECTED AND NORMAL FAMILY MEMBERS WITH SPLEENS INTACT. Each symbol indicates two individual values. an excellent test for separating these two groups. The high normal values are due in large part to the use of brilliant cresyl blue rather than the new methylene blue. Figure 2 shows the distribution of spherocyte scores for normal and affected subjects. There was a tendency to over-read normal smears. This was noticed by the examiners especially when five or more normal smears were studied in succession. Seventeen per cent of smears from the normal family members were read as showing spherocytes by two or more observers. However, only two smears from normal family members were read as positive by all three ob-

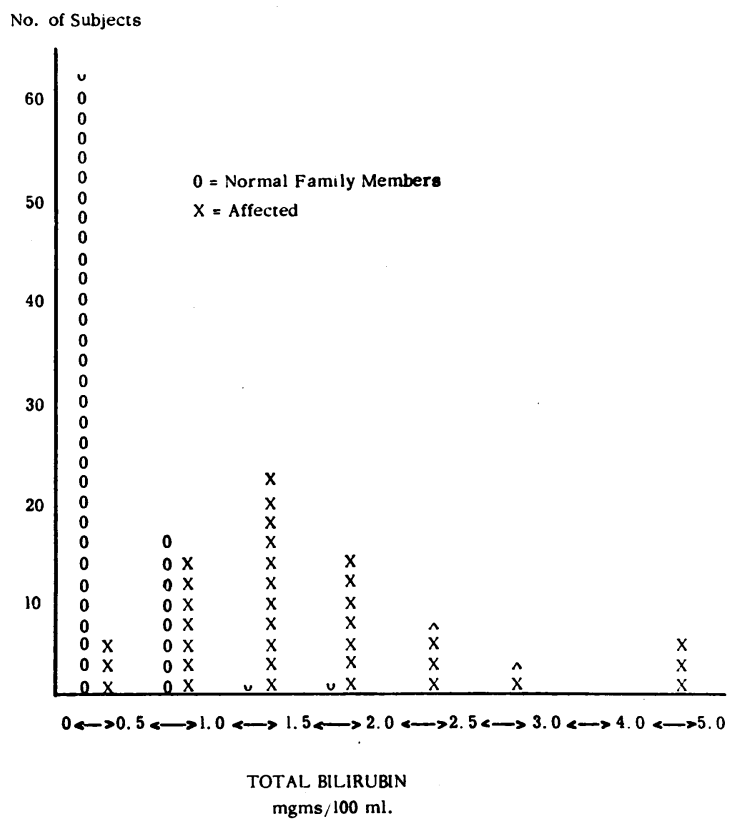

Fig. 3. Distribution of tOtAl Bilirubin values foR AFFECTED AND NORMAL FAMILY MEMBERS WITH SPLEENS INTACT. Half symbols indicate one individual.

servers. In one of these two (182308) the classification is uncertain. There was no tendency to under-read smears of affected individuals; 97 per cent of the affected had spherocytes according to the majority of observers.

Figure 3 shows a comparison of bilirubin values for normal and affected (total bilirubin was used, since statistical analysis showed high correlation between indirect and total bilirubin). Twentyeight per cent of affected fell within the normal range. Similarly for mechanical fragility (Figure 4) we see that 20 per cent of affected fall within the normal range. In Figure 5 the overlap be- 
tween affected and normal for hemoglobin values is nearly complete.

When values for reticulocytes, hemoglobin, bilirubin, and spherocytes were combined to form a discriminant, $D_{4}$, six discrepancies between $D_{4}$ and the physician's diagnosis were found among 159 family members with spleens. This is a 3.7 per cent discordance, using four tests. When all 13 tests were used to form a discriminant, Dc, three discrepancies between Dc and the physician's diagnosis were found among 110 family members. This is a 2.7 per cent discordance, using 13 tests.

\section{DISCUSSION}

The problem of diagnosis or ascertainment of hereditary spherocytosis for genetic analysis differs from classical medical diagnosis in several respects. The emphasis is on detection of disease in people who consider themselves well rather than proving that a given case of hemolytic anemia is typical of hereditary spherocytosis. The physician working with a sick patient may conveniently repeat a variety of laboratory tests until the diagnosis is certain. Ideally the medical geneticist would make a single quantitative measurement that would segregate the members of HS families into two groups. To date such a measurement has not become available. We must rely on a number of tests which describe the syndrome of hereditary spherocytosis. For genetic purposes we must make the group of tests large enough so that we can draw a correct conclusion from a single sample of blood, since daily fluctuations are expected for each test in the same individual and we are not likely to be able to re-study any significant number of these individuals.

What tests are available and how are they interpreted? Elevation of the reticulocyte count indicates increased red cell production. An increased indirect bilirubin suggests a hemolytic process, but increased values are found in other diseases-e.g., Gilbert's disease. Elevated osmotic fragility correlates with spherocytosis (23). Examination of the blood smear is the only way yet devised to demonstrate spherocytes, but it is the least objective of the available tests. Mechanical fragility is also an indicator of spherocytosis, and appears to be more sensitive than the osmotic fragility test (24). It is not specific, since ele-

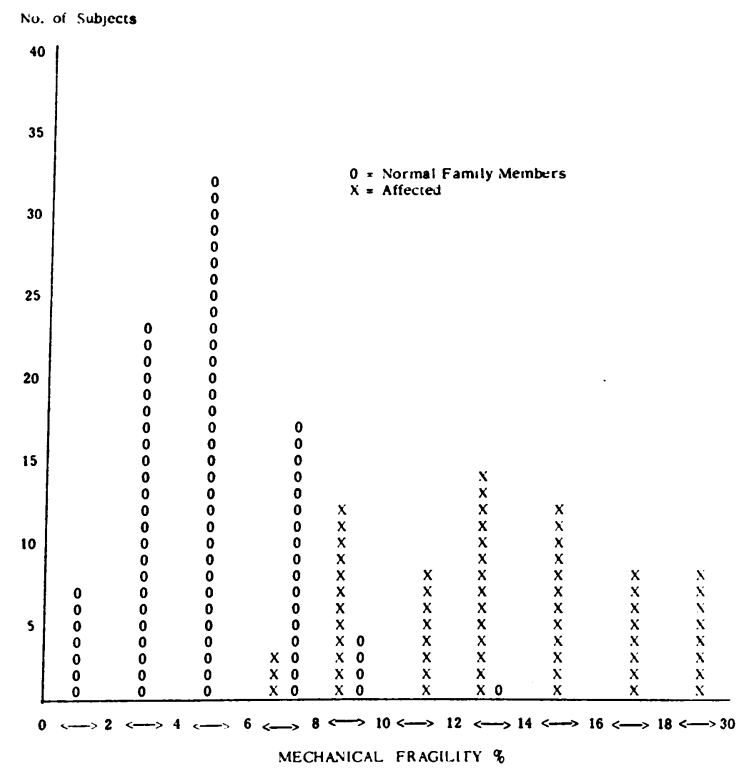

Fig. 4. Distribution of MEChanical FRAGility VAlUES FOR AFFECTED AND NORMAL FAMILY MEMBERS WITH SPLEENS INTACT. Each symbol indicates one subject.

No. of Subjects

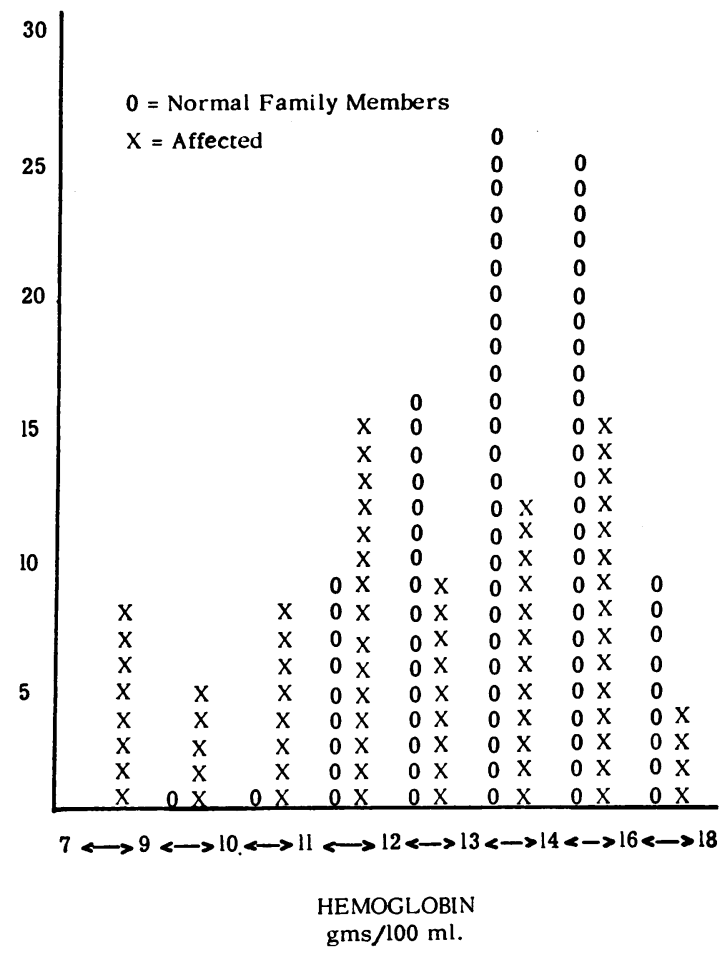

Fig. 5. Distribution of hemoglobin values for NORMAL AND AFFECTED FAMILY MEMBERS WITH SPLEENS INTACT. Each symbol indicates one subject. 
vated values are found in the presence of agglutinated cells or sickle cells (18). Autohemolysis is also an indicator of spherocytosis (Reference 13, p. 45) and is of importance to the diagnosis of the hereditary disease, since the high values found in this disease are markedly reduced by the addition of glucose (25). Red cells in acquired hemolytic anemias usually do not show significantly less autohemolysis with added glucose (20). Autohemolysis may be of further value in separating HS into two types, since it has been reported that addition of adenosine changes phosphorylated intermediates toward normal in some families and not in others (12).

Quantitative estimates of the correlations of these tests are given in Table VII. These estimates were made from the data of normal controls and classical cases of hereditary spherocytosis. They are not necessarily valid for normals alone, or for other hematologic diseases. The spherocyte score correlates most significantly with reticulocytes, mechanical fragility, 50 per cent osmotic fragility, and autohemolysis, in that order. The fragility tests are significantly and approximately equally correlated with each other; higher correlations are found within tests : 1 per cent osmotic fragility with 50 per cent osmotic fragility, and autohemolysis with autohemolysis plus glucose. The table also shows that a decrease in the mean corpuscular volume and an increase in the mean corpuscular hemoglobin concentration are correlated with increased fragility, as expected. Decreased hemoglobin and increased bilirubin are correlated with increased fragility.

Since the tests are highly intercorrelated, it is unlikely that all the tests are required to diagnose family members after the first case has been ascertained. There are two lines of evidence that establish this observation. The first is that comparison of the multiple correlation coefficients indicates little improvement in discrimination when a combination of all 13 tests is used instead of a combination of 4 tests. The multiple correlation coefficient is a discrimination ratio; that is, it gives a percentage value to the relative discriminative efficiency of various combinations of tests in separating the affected and normal groups. From the multiple correlation coefficients we can say that 78 per cent of the variability between the controls and 29 hand-picked affected is accounted for by the five fragility tests used to form $\mathrm{Dh}$ : autohemolysis, autohemolysis with glucose, osmotic fragility 1 per cent, osmotic fragility 50 per cent, and mechanical fragility. Eighty-eight per cent of the variability between the two groups is accounted for by four simple laboratory tests used to form $\mathrm{D}_{4}$ : hemoglobin, reticulocyte count, bilirubin, and spherocyte score. The multiple correlation coefficient for $D_{5}$, adding values for mechanical fragility to the four values used to form $\mathrm{D}_{4}$, is increased only slightly (to 90 per cent) over $\mathrm{D}_{4}$. The correlation coefficient for the complete discriminant using 13 tests is 91 per cent. Since the difference between the correlation coefficients for $\mathrm{D}_{4}$ and $\mathrm{Dc}$ is small, the difference in discriminative effectiveness between these two groups of tests is also small.

The second line of evidence is that there is no appreciable change in the discrepancy between the computer diagnosis and the physician's diagnosis when 13 tests are used instead of 4 . There was a 3.7 per cent discrepancy between $\mathrm{D}_{4}$ and the physician's diagnosis, and 2.7 per cent discrepancy between Dc and the physician's diagnosis. We conclude that there is little to be gained in doing more than these four tests on members of a family in which one typical case of hereditary spherocytosis has been found. These four tests in order of their importance to the discriminant $\mathrm{D}_{4}$ are: 1) spherocyte score, 2) reticulocyte count, 3) hemoglobin, 4) bilirubin. When the fragility tests and indices are added to these four simple hematologic tests there is a small improvement in discrimination. Most of this improvement can be accounted for by the mechanical fragility test alone. (Autohemolysis was decreased by addition of adenosine in all families studied. Consequently we have not considered autohemolysis with adenosine as a special test.)

Large-scale statistical evaluation of multiple laboratory data in medicine is relatively new. Zieve and Hill (26) in 1955 published an intensive study using the discriminant function to analyze the effectiveness of liver function tests in the diagnosis of liver disease. In 1959 Shephard and Turner (27) used the discriminant function to test multiple pulmonary function tests in a small group of patients with interstitial fibrosis. At least two studies $(28,29)$ have been made using both clinical and laboratory data to diagnose 20 or 
more hematologic diseases. One large study of nearly 6,000 patients was made by Brodman, Van Woerkom, Erdmann and Goldstein (30) in 1959 who used data from a symptom questionnaire as a means of diagnosing 60 diseases.

When many diagnostic possibilities are presented to the computer, the efficiency of selection is decreased. In the study by Brodman and coworkers (30) 48 per cent of computer diagnoses were correct. In the work of Lipkin and coworkers (29) 57 per cent of machine diagnoses were correct. We chose a relatively simple problem to present to the computer: separation of members of families with hereditary spherocytosis into affected and normal classes by compressing the variations within the two classes and thus minimizing the overlap. The machine was 97 per cent correct, with the physician's diagnosis as a standard, in carrying out this procedure.

Although we considerd the possibility that statistical analysis of multiple laboratory tests could result in detection of mildly affected cases that might be missed on inspection, we disagreed with the complete discriminant scores in three doubtful cases. Study of these three individuals gives some clues to the cause of the disagreements.

A 74 year old man, 168202, was considered by the physician to be affected on the basis of readily palpable spleen and a suggestive elevation of the autohemolysis. We feel that this conclusion is correct because subsequent study shows that a sister and a son have typical hereditary spherocytosis.

A 56 year old woman, 172302, was considered to be normal by the physician on the basis of normal bilirubin and reticulocytes, although the spherocyte score was 2 and other laboratory data were equivocal. We think this conclusion is correct because her medical history, genetic history, and physical examination were negative. She was the only normal spouse in the table of family members and was included because her husband (172303), by laboratory criteria, was unaffected; however, genetic studies indicated plainly that he was the genetic carrier. (This problem of unaffected carriers will be discussed in greater detail in a subsequent paper.)

A 26 year old man, 182308, was considered to be normal by the physician because the bilirubin and reticulocytes were normal. $\mathrm{He}$ was considered affected by statistical discrimination on the basis of a spherocyte score of 3 . The physician's diagnosis was made before the statistical analysis was known. In restrospect, on the basis of the importance now attached to a spherocyte score of 3 , the physician would have considered this man to be affected.
The physician's judgment in the first case was based partially on a palpable spleen, information not used by the computer. In the second and third cases the physician's evaluation of the laboratory data, particularly the spherocyte score, difered from the computer analysis. We are not certain which evaluation is correct.

Obviously the criteria chosen by the physician and by the computer were not identical. Nevertheless there was good agreement between the physician's diagnosis and statistical evaluation by the computer. This good agreement indicates that the computer did not ascertain more mildly affected cases than could be diagnosed by the physician. We would predict from this that our genetic data would be similar to the data of other contemporary workers, assumed that sufficient laboratory values were available to them. This proved true.

We found an incidence of $0.51 \pm 0.042$ with a slight excess of males (0.56). The excess of affected males is not significant (14) except when pooled with the data of Young and colleagues (10), Race (8), and Abrams and Battle (9). The incidence of affected is higher than Race or Young and colleagues found, but the difference is not significant. Although criteria of ascertainment in work previously cited differed from ours or may not have been stated, we infer that the judgment of the physicians was based on enough data to draw conclusions similar to ours. We conclude that this method of discriminating normal from affected family members does not significantly increase the number of mildly affected cases that are detected, since the genetic data are statistically homogeneous with those of Race, Young and colleagues, and Abrams and Battle.

This study provides us with a quantitative determination of tests needed to diagnose family members correctly. Ninety-seven per cent of the cases was correctly classified by tests arbitrarily chosen as the most generally available: the smear, reticulocyte count, hemoglobin, and bilirubin. This does not discredit the fragility tests, since they contribute a significant amount of information when used independent of other measurements. But they have proved to be uneconomical except in diagnosing the primary case in a family and other isolated cases of hemolytic anemia. The formula for the discriminant $\mathrm{D}_{4}$ is considered 
to be applicable to other family studies of hereditary spherocytosis, provided that controls are used to determine distribution of discriminant scores for normal subjects in other laboratories.

Data from this study can practicably be pooled with data from Race (8), Young and co-workers (10), and Abrams and Battle (9). With this larger volume of data we are able to investigate problems of fitness, incidence, and mutation that are not yet solved for hereditary spherocytosis.

\section{SUMMARY}

We have studied 181 members of 26 families with hereditary spherocytosis. Means and standard deviations for 12 tests have been calculated for four groups: controls, normal family members, affected family members with spleens intact, and affected family members after splenectomy.

Correlations of tests individually indicated many significant interrelationships. Correlations of groups of tests indicated that 4 tests (spherocyte score, reticulocyte count, hemoglobin, and bilirubin) accounted for 88 per cent of the variability between normal subjects and classical cases of hereditary spherocytosis, whereas 13 tests accounted for 91 per cent of the variability. Addition of mehanical fragility to the four tests mentioned accounted for 90 per cent of the variability between the two classes.

Discriminant scores for 4 tests $\left(D_{4}\right)$ and 13 tests (Dc) were made for family members and normal controls. When these were compared with the physician's evaluation, there was a 3.7 per cent discrepancy between the physician and $\mathrm{D}_{4}$, and a 2.7 per cent discrepancy between the physician and Dc.

Diagnosis of family members with hereditary spherocytosis does not ordinarily require more than four tests after the initial case has been studied.

Genetic data from this study give a higher incidence of affected than do other contemporary investigations, but the difference is not statistically significant.

\section{ACKNOWLEDGMENTS}

We are indebted to Miss Frances Morris, Mrs. Kay Strutz, Mrs. Virginia Loy, and Miss Mary Muckerheide for technical assistance, and to Mrs. Marie Rosenblum and Mrs. Joan Owens for secretarial assistance.

\section{REFERENCES}

1. Weisman, R., Jr., Ham, T. H., Hinz, C. F., Jr., and Harris, J. W. Studies of the role of the spleen in the destruction of erythrocytes. Trans. Ass. Amer. Phycns 1955, 68, 131.

2. Young, L. E., Platzer, R. F., Ervin, D. M., and Izzo, M. J. Hereditary spherocytosis. II. Observation on the role of the spleen. Blood 1951, 6, 1099.

3. Prankerd, T. A. J., Altman, K. I., and Young, L. E. Abnormalities of carbohydrate metabolism of red cells in hereditary spherocytosis. J. clin. Invest. 1955, 34, 1268.

4. Prankerd, T. A. J. Studies on the pathogenesis of haemolysis in hereditary spherocytosis. Quart. J. Med. 1960, 29, 199.

5. Greenwalt, T. J., and Ayers, V. E. The phosphate partition of the erythrocytes of normal newborn infants and of infants with hemolytic disease. J. clin. Invest. 1956, 35, 1404.

6. Allison, A. C., Kates, M., and James, A. T. An abnormality of blood lipids in hereditary spherocytosis. Brit. med. J. 1960, 2, 1766.

7. Campbell, J. M. H., and Warner, E. C. Hereditary in acholuric jaundice. Quart. J. Med. 1925-26, 19, 333.

8. Race, R. R. On the inheritance and linkage relations of acholuric jaundice. Ann. Eugen. (Lond.) 1942, 11,365 .

9. Abrams, M., and Battle, J. D., Jr. A genetic study in hereditary spherocytosis. Amer. J. hum. Genet. 1952, 4, 350.

10. Young, L. E., Izzo, M. J., and Platzer, R. F. Hereditary spherocytosis. I. Clinical, hematologic and genetic features in twenty-eight cases, with particular reference to the osmotic and mechanical fragility of incubated erythrocytes. Blood 1951, 6, 1073.

11. Lawrence, J. A genetic study of a family with hereditary spherocytosis (H.S.) and hyperuricemia. Clin. Res. 1960, 8, 211.

12. Young, L. E. Observations on inheritance and heterogeneity of chronic spherocytosis. Trans. Ass. Amer. Phycns 1955, 68, 141.

13. Dacie, J. V. The Haemolytic Anaemias, Congenital and Acquired, 2nd ed. London, Churchill, 1960 , part I.

14. Morton, N. E., MacKinney, A. A., Jr., Schilling, R. F., Kosower, N. S., and Gray, M. Genetics of spherocytosis. Amer. J. hum. Genet. In press. 1962, 14 (June).

15. MacKinney, A. A., Jr., and Schilling, R. F. Family studies of hereditary spherocytosis. To be published.

16. Wintrobe, M. M. Clinical Hematology, 4th ed. Philadelphia, Lea \& Febiger, 1956, p. 366.

17. Ducci, H., and Watson, C. J. The quantitative determination of the serum bilirubin with special reference to the prompt-reacting and the chloro- 
form-soluble type. J. Lab. clin. Med. 1945, 30, 293.

18. Shen, S. C., Castle, W. B., and Fleming, E. M. Experimental and clinical observations on increased mechanical fragility of erythrocytes. Science 1944, 100, 387.

19. Emerson, C. P., Jr., Shen, S. C., Ham, T. H., Fleming, E. M., and Castle, W. B. Studies on the destruction of red blood cells. IX. Quantitative methods for determining the osmotic and mechanical fragility of red cells in the peripheral blood and splenic pulp; the mechanisms of increased hemolysis in hereditary spherocytosis (congenital hemolytic jaundice) as related to the functions of the spleen. A.M.A. Arch. intern. Med. 1956, 97, 1.

20. Young, L. E., Izzo, M. J., Altman, K. I., and Swisher, S. N. Studies on spontaneous in vitro autohemolysis in hemolytic disorders. Blood 1956, 11, 977.

21. Fisher, R. A. The use of multiple measurements in taxonomic problems. Ann. Eugen. (Lond.) 1936, 7, 179.

22. Fisher, R. A. The statistical utilization of multiple measurements. Ann. Eugen. (Lond.) 1938, 8, 376.

23. Haden, R. L. The mechanism of the increased fragility of the erythrocytes in congenital hemolytic jaundice. Amer. J. med. Sci. 1934, 188, 441.
24. Schaub, F., and Maier, C. Zur klinischen Bedeutung der mechanischen Resistenz der roten Blutkörperchen. Acta haemat. (Basel) 1956, 15, 90.

25. Selwyn, J. G., and Dacie, J. V. Autohemolysis and other changes resulting from the incubation in vitro of red cells from patients with congenital hemolytic anemia. Blood 1954, 9, 414.

26. Zieve, L., and Hill, E. An evaluation of factors influencing the discriminative effectiveness of a group of liver function tests. III. Relative effectiveness of hepatic tests in cirrhosis. Gastroenterology 1955, 28, 785.

27. Shephard, R. J., and Turner, M. E. On the probability of correct diagnosis by pulmonary function tests. Thorax 1959, 14, 300.

28. Lipkin, M., and Hardy, J. D. Mechanical correlation of data in differential diagnosis of hematological diseases. J. Amer. med. Ass. 1958, 166, 113.

29. Lipkin, M., Engle, R. L., Jr., Davis, B. J., Zworykin, V. K., Ebald, R., Sendrow, M., and Berkley, C. Digital computer as aid to differential diagnosis. A.M.A. Arch. intern. Med. 1961, 108, 56.

30. Brodman, K., van Woerkom, A. J., Erdmann, A. J., Jr., and Goldstein, L. S. Interpretation of symptoms with a data-processing machine. A.M.A. Arch. intern. Med. 1959, 103, 776. 